\title{
Genome-wide analysis identifies cis-acting elements regulating mRNA polyadenylation and translation during vertebrate oocyte maturation
}

\author{
FEI YANG, ${ }^{1,2}$ WEI WANG, ${ }^{1,2}$ MURAT CETINBAS, ${ }^{1,2}$ RUSLAN I. SADREYEV, ${ }^{1,3}$ and MICHAEL D. BLOWER ${ }^{1,2}$ \\ ${ }^{1}$ Department of Molecular Biology, Massachusetts General Hospital, Boston, Massachusetts 02114, USA \\ 2Department of Genetics, Harvard Medical School, Boston, Massachusetts 02115, USA \\ ${ }^{3}$ Department of Pathology, Massachusetts General Hospital and Harvard Medical School, Boston, Massachusetts 02115, USA
}

\begin{abstract}
Most cells change patterns of gene expression through transcriptional regulation. In contrast, oocytes are transcriptionally silent and regulate mRNA poly(A) tail length to control protein production. However, the genome-wide relationship of poly(A) tail changes to mRNA translation during vertebrate oocyte maturation is not known. We used Tail-seq and polyribosome analysis to measure poly(A) tail and translational changes during oocyte maturation in Xenopus laevis. We identified large-scale poly(A) and translational changes during oocyte maturation, with poly(A) tail length changes preceding translational changes. Proteins important for completion of the meiotic divisions and early development exhibited increased polyadenylation and translation during oocyte maturation. A family of U-rich sequence elements was enriched near the polyadenylation signal of polyadenylated and translationally activated mRNAs. We propose that changes in mRNA polyadenylation are a conserved mechanism regulating protein expression during vertebrate oocyte maturation and that these changes are controlled by a spatial code of cis-acting sequence elements.
\end{abstract}

Keywords: oocyte; polyadenylation; translation

\section{INTRODUCTION}

Accurate regulation of gene expression is critical for development and cell-fate specification. In most cell types, changes in gene expression are controlled at the level of transcription. However, oocytes are transcriptionally silent and changes in gene expression are controlled by posttranscriptional mechanisms (Mendez and Richter 2001). Oocytes complete premeiotic DNA replication and recombination, then arrest in prophase of meiosis I (Hassold and Hunt 2001). Upon receiving a hormonal signal, oocytes exit the prophase arrest and enter the meiotic divisions. In vertebrates, oocytes undergo nuclear envelope breakdown (also called germinal vesicle breakdown, GVBD), segregate homologous chromosomes at meiosis I, extrude a polar body, and arrest at metaphase of meiosis II until fertilization. Completion of these dramatic morphological events requires the coordinated synthesis of many different proteins. Aneuploidy resulting from inaccurate chromosome segregation during the meiotic divisions is a leading cause of birth defects in humans (Nagaoka et al.

Corresponding author: blower@molbio.mgh.harvard.edu

Article is online at http://www.rnajournal.org/cgi/doi/10.1261/rna. 073247.119 .
2012), therefore understanding gene expression changes during meiotic maturation is critical to understanding the cause of aneuploidy.

Gene expression during oocyte maturation is controlled by cytoplasmic polyadenylation of stored mRNAs (Mendez and Richter 2001). During transcription, mRNAs receive a long poly(A) tail in the nucleus that facilitates mRNA export. In oocytes, the long poly(A) tails of most mRNAs are removed in the cytoplasm, which triggers "masking" or translational silencing (Weill et al. 2012). As oocytes, mature phosphorylation cascades unmask stored mRNAs leading to rapid polyadenylation and polyadenylation-dependent translational activation (Richter and Lasko 2011). Cytoplasmic polyadenylation is facilitated by a cis-acting sequence element termed the cytoplasmic polyadenylation element (CPE, UUUUAU) which is bound by the CPEB protein (Richter 2007). CPEB and CPEs are important for polyadenylation and polyadenylation-dependent

(C) 2020 Yang et al. This article is distributed exclusively by the RNA Society for the first 12 months after the full-issue publication date (see http://rnajournal.csh/p.org/site/misc/terms.xhtml). After 12 months, it is available under a Creative Commons License (Attribution-NonCommercial 4.0 International), as described at http:// creativecommons.org/licenses/by-nc/4.0/. 
translation of the mos and cyclin B mRNAs during oocyte maturation in Xenopus laevis (Sheets et al. 1994). Importantly, cytoplasmic polyadenylation is critical for oocyte maturation as blocking this process completely blocks progress into meiosis (Kuge and Inoue 1992).

Most of our current knowledge of the mechanisms of cytoplasmic polyadenylation comes from detailed studies of single transcripts. Until recently it has not been possible to measure the length of poly(A) tails at a genome-wide scale. In the past 5 years, important technical advances have provided the first genome-scale views of changes in transcript polyadenylation (Chang et al. 2014; Subtelny et al. 2014). Work from the Bartel and Kim groups have adapted Illumina sequencing to measure poly(A) tail length in several experimental systems (Chang et al. 2014; Kronja et al. 2014; Subtelny et al. 2014; Eichhorn et al. 2016; Park et al. 2016). This work has shown that poly $(A)$ tail lengths are carefully regulated during early development in Drosophila, Xenopus, and zebrafish. In addition, recent work in Drosophila has shown that poly $(\mathrm{A})$ tail length regulation is important for controlling translation during Drosophila oogenesis (Eichhorn et al. 2016; Lim et al. 2016) and transcript stability in mouse oocytes (Morgan et al. 2017). Interestingly, poly(A) tail length is well correlated with translational activity during oogenesis and early development, but becomes uncoupled in somatic cells and at the midblastula transition (Subtelny et al. 2014). These recent studies have provided important genome-wide insight into the regulation of poly $(A)$ tail length during early development and revealed that poly(A) tail regulation is a major mechanism controlling gene expression. However, these studies have not determined how poly $(A)$ tail lengths are regulated during oocyte maturation in a vertebrate system.

In this work we used multiple genome-wide approaches to measure poly $(A)$ tail length and mRNA translation in $X$. laevis oocytes during oocyte maturation. Our results show that poly(A) tail length regulation is a major mechanism controlling mRNA translation in vertebrate oocyte maturation. Additionally, our work provides insight into cis-acting sequence elements that control polyadenylation and translation. Our genome-scale approach provides insight into the mechanisms of gene expression control in oocytes and into new proteins important for the completion of the meiotic divisions.

\section{RESULTS}

\section{Poly(A) tail measurement during oocyte maturation}

To measure changes in poly(A) tail length during Xenopus laevis oocyte maturation, we implemented the Tail-seq method in our laboratory (Chang et al. 2014). We synthesized spike-in sequences with defined poly(A) lengths $(0,8,16,32,64,90,118 \mathrm{nt})$, converted these sequences into an Illumina library, and sequenced the library. We initially tested the ability of the Illumina base-calling software to correctly infer the length of poly(A) stretches in each sample. Consistent with previous results we found that the Illumina base-calling software performed poorly on poly(A) sequences longer than 32 nt (Fig. 1A; Chang et al. 2014). We then created a custom algorithm to measure poly $(A)$ length based on the detection of the boundary between the poly $(A)$ sequence and the rest of the transcript. In brief, as opposed to the Illumina fastq sequence files, we used raw fractions of fluorescent intensity corresponding to each base $(A, T, G, C)$ within a 5 -nt sliding window around every position of the sequencing read, and detected the shift of these frequencies between adjacent sequence positions (see Materials and Methods for details). Our custom poly(A) calling algorithm produced much more accurate poly(A) length determinations on the synthetic spike-in library (Fig. 1B).

To measure genome-wide changes in poly $(A)$ tail length, we harvested immature oocytes from $X$. laevis females and induced oocyte maturation by the addition of progesterone. We measured meiotic progression by both GVBD and cyclinB:cdk kinase activity. Consistent with previous studies (Mendez and Richter 2001), oocytes progressed through meiosis in a stereotypical pattern with two waves of elevated cyclinB:colk activity representing metaphase of meiosis I and metaphase of meiosis II (Fig. 1C). We collected five time points during meiotic progression from two different frogs: untreated (noPG), 90 min after progesterone addition (PG90), meiosis I (MI), interkinesis (Int.), and meiosis II (MII). Oocytes from each stage were collected in parallel for analysis of both poly(A) tail length and protein translation by sucrose density gradient. We used a modified PAT method (Harrison et al. 2015) to append a 3' linker to the end of all polyadenylated mRNAs and converted these mRNAs into a sequencing library that includes intact poly $(A)$ tails. We sequenced libraries from five time points from biological replicates using paired-end sequencing: a $250 \mathrm{nt}$ read that sequenced across the poly $(\mathrm{A})$ tail and a $50 \mathrm{nt}$ read to determine the transcript of origin. We analyzed genes with at least 50 reads in all five time points in both biological replicates (5690 genes, Supplemental Tables S1, S2), which is a stringent read cutoff originally implemented in the first measurement of poly(A) tail lengths by sequencing (Subtelny et al. 2014). We found that biological replicates exhibited similar behavior using principal component analysis (PCA) (Fig. 1D), and that the major difference was between samples with inactive (noPG, PG90) and active cyclinB:cdk (MI, Int., MII). We found smaller, reproducible differences between samples as oocytes progressed through the meiosis divisions on PC2. Analysis of the average poly $(A)$ tail length of transcripts revealed large-scale changes at different stages of meiosis (Fig. $1 E)$. Untreated oocytes exhibited a peak poly(A) tail length of $\sim 40 \mathrm{nt}$ that was unchanged $90 \mathrm{~min}$ after progesterone 

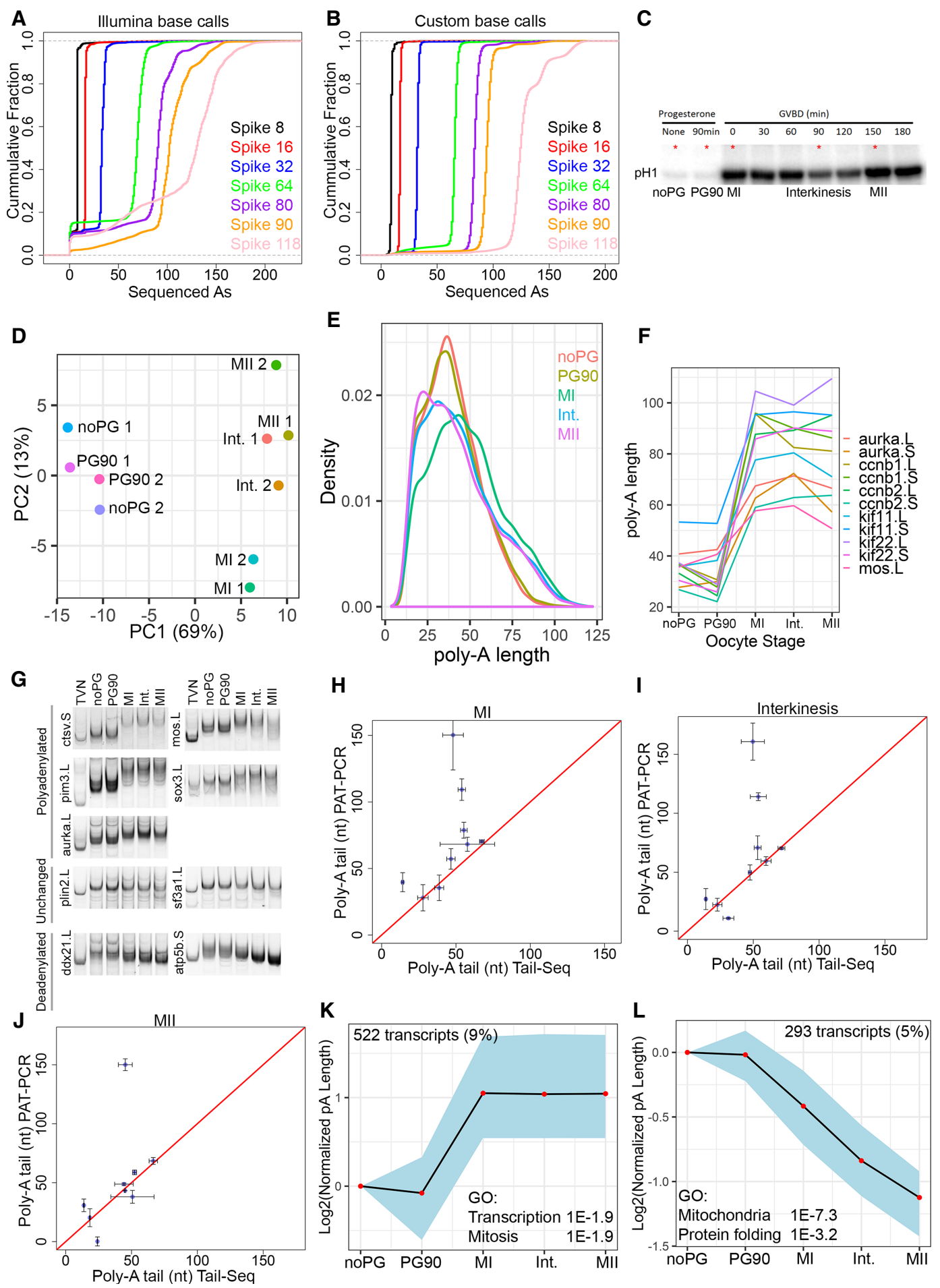

FIGURE 1. Measurement of poly(A) tail changes during oocyte maturation. (A) Synthetic spike sequences containing various lengths of A homopolymers were sequenced using an Illumina miSeq. Cumulative distribution plot shows the poly(A) lengths as determined by the Illumina base-calling software. (B) A custom algorithm was developed to more accurately determine the length of poly(A) sequences from raw Illumina sequencing files. Cumulative distribution plot shows the distribution of poly(A) lengths determined by our custom software on the same sequencing data presented in A. (C) Histone $\mathrm{H} 1$ kinase assays of oocytes as they progress through meiotic maturation. Red asterisk indicates time points that were used for Tail-seq and polysome analysis. (D) Tail-seq measurements from two biological replicates were analyzed using principal components analysis. PCA plot shows the relationship between different time points and different biological replicates. (E) Histogram showing measured poly $(A)$ tail lengths at five time points spanning meiotic maturation. Curves are the average of two replicates. $(F)$ Average measured poly $(A)$ tail lengths for mRNAs previously reported to be polyadenylated during oocyte maturation. In X. laevis many genes have paralogs which are indicated by a .L and .S designation. (G) PAT assay was used to analyze poly(A) tail length of the indicated transcripts during oocyte maturation. Similar results were observed in an additional biological replicate (not shown). Poly(A) tail predictions from our Tail-seq data are indicated beside the gene name. $(H-J)$ Scatterplots comparing poly(A) tail lengths measured by Tail-seq and the PAT assay at MI, Interkinesis, and MII. (K) STEM cluster of transcripts exhibiting increased polyadenylation during oocyte maturation. Enriched GO terms are indicated on the plot. (L) STEM cluster of deadenylated transcripts and enriched GO terms. 
addition. However, as oocytes entered Ml we observed a large-scale polyadenylation of most mRNAs followed by global deadenylation in Int. and MII (Fig. 1E), consistent with the observation that histone B4 and setd8a mRNAs are polyadenylated then deadenylated during oocyte maturation (de Moor and Richter 1997; Curanovic et al. 2013). Interestingly, a substantial number of mRNAs retained long poly(A) tails throughout meiosis. To determine if our Tailseq measurements accurately reflect known patterns of mRNA polyadenylation, we analyzed the poly $(A)$ tail lengths of several mRNAs previously shown to be polyadenylated during meiosis (Fig. 1F; Sheets et al. 1994; Eliscovich et al. 2008). We found that all previously characterized mRNAs exhibited dramatic poly(A) tail increases during meiosis. Additionally, we used the PAT assay to measure poly $(A)$ tail changes in mRNAs with increased, decreased, and unchanged poly(A) tails (Fig. 1I). In all tested mRNAs our sequencing results correctly predicted the direction of poly $(A)$ tail length change. For some mRNAs with very long poly(A) tails, we found that the PAT assay reported longer poly(A) lengths than Tail-seq (Fig. $1 \mathrm{H}-J$ ), consistent with a recent study that found that Tail-seq underestimates long poly(A) tails (Morgan et al. 2017). Taken together, these results demonstrate that Tail-seq accurately captures changes in transcript poly(A) tail length during $X$. laevis oocyte meiosis.

To determine if groups of transcripts exhibited coordinated polyadenylation behavior, we used STEM software (Ernst and Bar-Joseph 2006) to cluster transcripts. STEM software uses a novel clustering algorithm to identify coordinately regulated transcripts in short time series experiments. We identified two major clusters of transcripts that exhibited increased and decreased adenylation during meiosis (Fig. 1K,L). Five hundred and twenty-two transcripts ( $9 \%$ of measured) increased poly $(A)$ tail length at least twofold during meiosis. Gene Ontology analysis of these transcripts using DAVID software (Huang da et al. 2009) demonstrated that transcripts coding for transcription and mitosis-related proteins were enriched in this set (Supplemental Table 3). Of note, several cyclin proteins (A1, B1, B2), proteins involved in regulation of kinetochore:microtubule attachment (Ndc80, Kif18b), and proteins involved in spindle assembly (Kif11, SPDL1, Aurora-A) exhibited increased polyadenylation, suggesting that polyadenylation of these mRNAs may promote their translation in order to complete the meiotic divisions. Many regulators of sister chromatid cohesion (Mau2, Esco2, Sgo1, Securin) are also polyadenylated during oocyte maturation. Interestingly, the cohesin complex is not loaded onto chromatin during meiosis in mice (Revenkova et al. 2010; Tachibana-Konwalski et al. 2010), suggesting that Mau2 and Esco2 may be polyadenylated during meiosis in order to produce proteins for early development rather than completion of meiosis. Additionally, 293 transcripts ( $\sim 5 \%$ of measured) exhibited at least twofold decrease in poly $(A)$ tail length. $G O$ analysis demonstrated that transcripts encoding mitochondrial and protein folding proteins were enriched in this cluster (Fig. 1L; Supplemental Table 4). A wide spectrum of mitochondrial proteins were deadenylated (electron transport chain, ribosomal proteins, general metabolic enzymes) suggesting that a large scale down-regulation of mitochondrial growth occurs during oocyte maturation. Taken together our results demonstrate that oocytes execute global changes in mRNA polyadenylation during oocyte maturation, which may be important for producing proteins for completion of meiosis and early embryonic divisions.

\section{Transcript degradation during oocyte maturation}

In most cell types RNA deadenylation is rapidly followed by RNA degradation by the $3^{\prime}-5^{\prime}$ exonuclease (Schoenberg and Maquat 2012). However, oocytes contain large stores of maternal RNAs that are required for early development and it is not clear if oocytes possess active RNA degradation pathways (Vastenhouw et al. 2019). To measure RNA stability during the course of oocyte maturation, we monitored the levels of all transcripts in total RNA samples as oocytes progressed through meiosis. At early time points, we observed no reproducible changes in transcript abundance (Fig. 2A,B). At later time points, we observed reproducible changes in mRNA abundance, with the most obvious changes being RNA degradation (Fig. 2D). STEM clustering identified 267 transcripts ( $2 \%$ of measured) as decreasing in abundance by at least twofold in two biological replicates (Fig. 2E). GO analysis demonstrated that degraded transcripts were enriched for ribosomal and mitochondrial protein mRNAs (Supplemental Table S5). Our GO analysis is consistent with a study that used Click chemistry to study large-scale changes in poly(A) tail length in Xenopus oocytes (Curanovic et al. 2013). We tested RNA stability for nine transcripts that were predicted to be degraded in additional biological replicates by Q-RT-PCR. We observed dramatic degradation in 8/9 tested transcripts (Fig. 2G). We also tested 10 transcripts that exhibited apparent increases in our sequencing data but were unable to reproduce these changes using Q-RT-PCR. This result is likely caused by the variability in observed transcript increases between different biological replicates (Fig. 2A-D). To determine if there is a correlation between RNA deadenylation and degradation, we compared normalized poly(A) tail lengths at MII to RNA stability measures for all mRNAs that exhibited consistent mRNA degradation. Interestingly, we found no clear relationship between RNA deadenylation and stability (Fig. 2F). Many RNAs with long poly(A) tails were effectively degraded, while others with short poly $(A)$ tails were also degraded. These results show that oocytes execute a reproducible program of RNA degradation during oocyte maturation. 
A

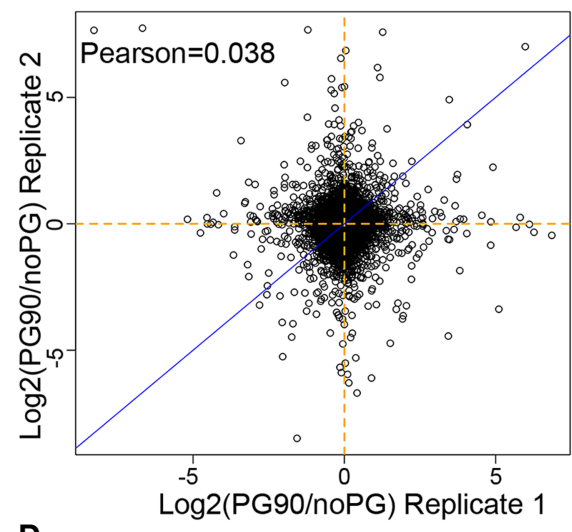

D

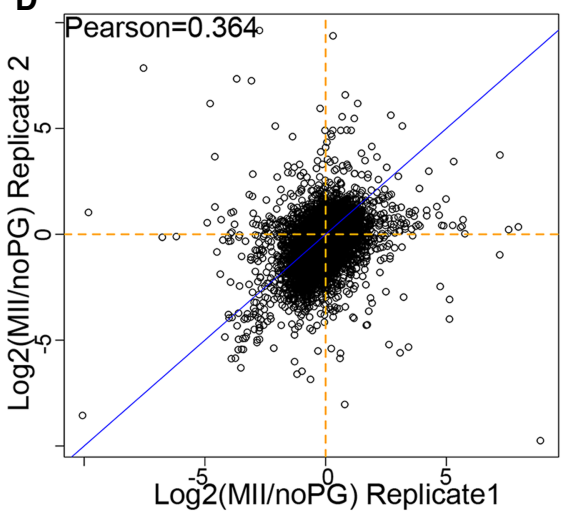

G

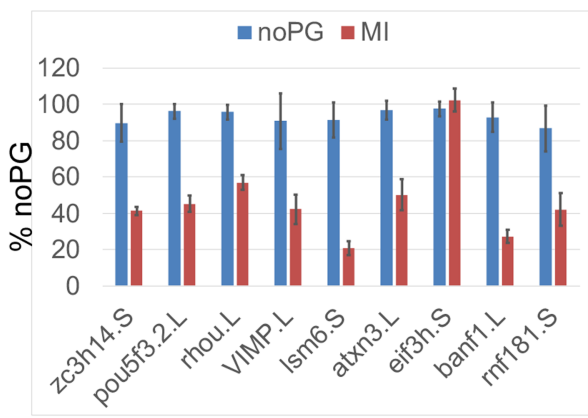

B

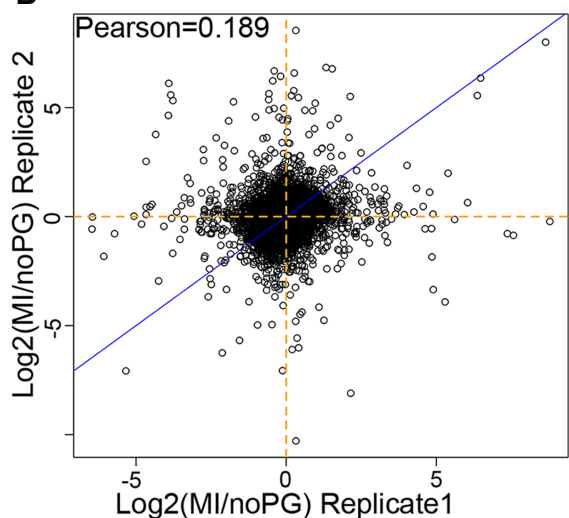

E
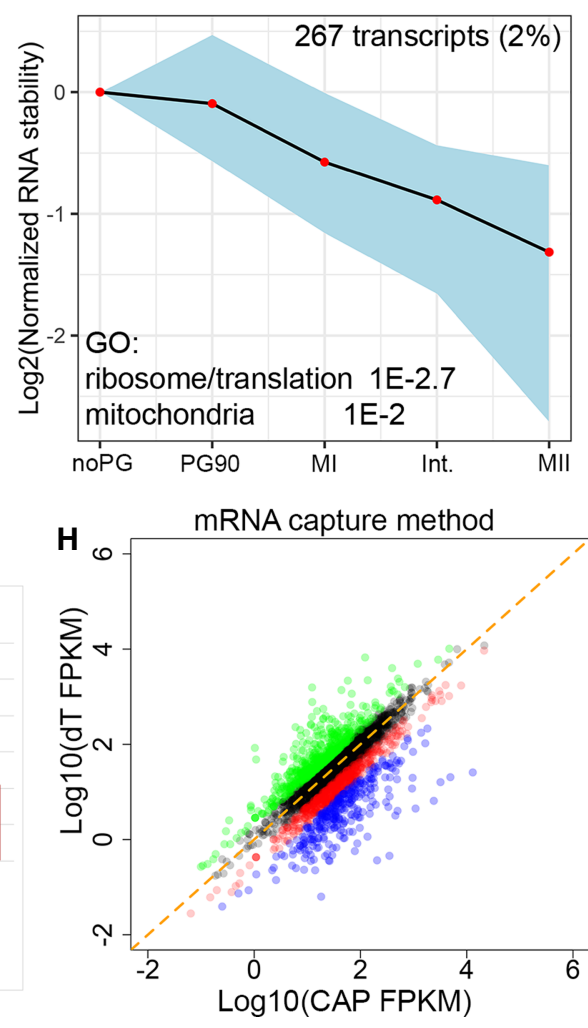

C

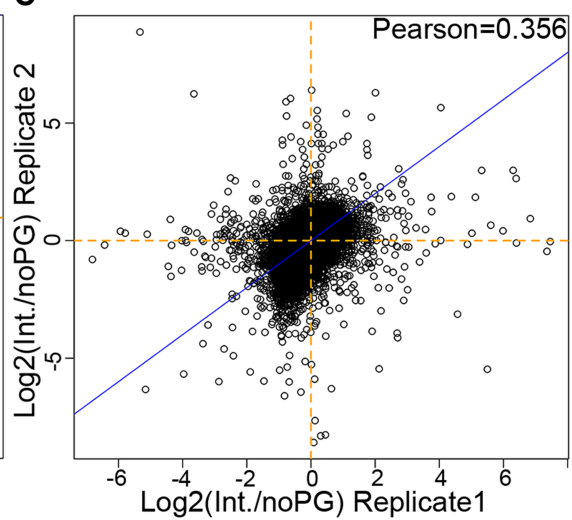

F

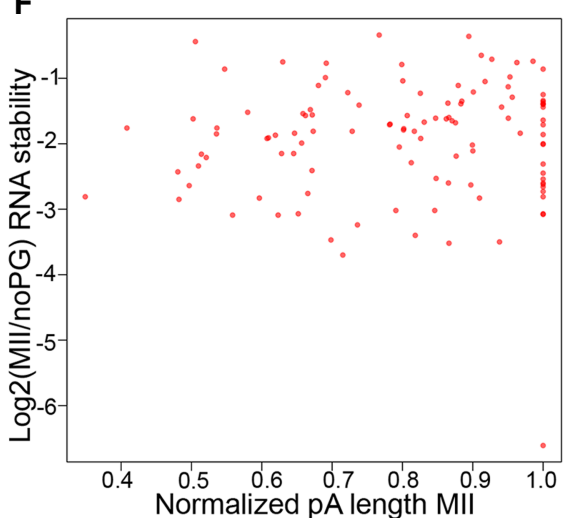

I

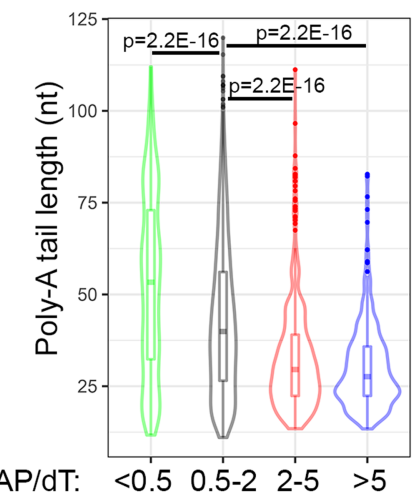

FIGURE 2. RNA degradation during oocyte maturation. RNA-seq reads from polysome gradients were pooled to create "total RNA" samples for analysis of transcript stability during oocyte maturation. (A-D) Scatterplots comparing two biological replicates of changes in RNA abundance during oocyte maturation. (E) STEM software was used to identify clusters of transcripts that were up- or down-regulated during oocyte maturation. One cluster of degraded transcripts was identified. GO terms enriched in this set of transcripts are indicated on plot. (F) Scatterplot comparing change in RNA stability for degraded transcripts (from $E$ ) to normalized measured poly(A) tail length. (G) Q-RT-PCR was used to measure the stability of nine transcripts predicted to be degraded from two additional biological replicates. We also tested several transcripts that were predicted to be increased during oocyte maturation and were unable to confirm any changes in transcript levels. Error bars indicate standard deviation after normalization to an unchanged transcript. (H) Comparison of RNA-seq abundance of mRNAs captured by oligo(dT) or Cap-capture from Blower et al. (2013). Transcripts are colored by ratio of FPKM between dT and Cap-capture libraries. (I) Violin plots of poly(A) tail length for each class of transcripts from $\mathrm{H}$. CAP/dT ratios are indicated below the plot. P-values are the result of a Wilcoxon rank-sum test.

Previous work has shown that poly(A) tail length can affect the efficiency of mRNA recovery by oligo(dT) selection (Beilharz and Preiss 2007; Blower et al. 2013). To directly compare poly(A) tail length to oligo(dT) pulldown efficiency, we compared our Tail-seq measurements to sequencing data that we previously generated from mature
Xenopus eggs using mRNA capture by oligo(dT) and capture by the 5' 7-methyl-G cap (Bajak and Hagedorn 2008; Blower et al. 2013). For our previous sequencing libraries we compared transcript levels (FPKM) in the oligo(dT) library to the Cap-capture library and observed that some transcripts were overrepresented in each of the capture 
methodologies (Fig. 2H). We separated these mRNAs into different groups based on the ratio of reads in dT compared to Cap-capture (Fig. $2 \mathrm{H}, \mathrm{I})$ and examined poly(A) tail length for each group (Fig. 2I). We found that mRNAs that were overrepresented in the oligo(dT) libraries had significantly longer poly $(\mathrm{A})$ tails than mRNAs equally present in both libraries. Additionally, mRNAs overrepresented in Cap-capture libraries had significantly shorter poly(A) tails than mRNAs equally present in both libraries (Fig. 2I). These comparisons confirm that extreme changes in poly $(A)$ tail length can influence transcript recovery in sequencing libraries prepared using oligo(dT) selection.

\section{mRNA translation analysis during oocyte maturation}

In Drosophila oocytes and several early embryonic systems, mRNA poly $(A)$ tail length is well correlated with changes in mRNA translation (Subtelny et al. 2014; Eichhorn et al. 2016; Lim et al. 2016). Additionally, pioneering work in $X$. laevis oocytes showed that cytoplasmic polyadenylation is necessary to initiate translation of the mos and cyclin B1 mRNAs (Sheets et al. 1994; de Moor and Richter 1997; Piqué et al. 2008). To globally determine how changes in mRNA polyadenylation relate to mRNA translation during vertebrate oocyte maturation, we measured mRNA association with polysomes from the same samples that we used to perform Tail-seq. To determine the efficiency of mRNA translation, we separated oocyte extracts on sucrose density gradients and isolated fractions corresponding to: mRNP/40/60S, monosomes, and polysomes. We prepared Illumina libraries from each fraction for each stage of oocyte maturation and calculated the percentage of each mRNA present in the polysome gradient fraction after normalization. We measured the polysome association of 6497 mRNAs using this approach (Supplemental Table S6). PCA analysis demonstrated that different stages of oocyte maturation were reproducibly well separated by PC1, while PC2 primarily reflected differences between biological replicates (Fig. 3A). A comparison of changes on mRNA polysome occupancy between different biological replicates demonstrates a positive correlation between $\mathrm{MI}$, Interkinesis, and MII (Fig. 3B-E). The biological variation present in this experiment reduces our ability to detect small changes in translation, but the resulting data set represents robust translational changes that occur during oocyte maturation. This variation may result from the fact that we used wild-type outbred Xenopus frogs for these experiments and there is well-known variability between Xenopus individuals (Hannak and Heald 2006; Harland and Grainger 2011) Analysis of the average polysome fraction of all transcripts revealed general features of mRNA translation during oocyte maturation (Fig. 3F). We observed a progressive loss of transcripts from the polysome fraction as oocytes progressed through meiosis, consistent with a general repression of translation during M phase (Prescott and Bender 1962; Pyronnet et al. 2001; Tanenbaum et al. 2015). Interestingly, we also observed a long tail of transcripts that exhibited increased polysome association during oocyte maturation (Fig. 3F). To verify that changes in mRNA polysome association accurately reflect changes in protein production, we analyzed eight candidate proteins that showed increased polysome association by quantitative western blot from additional biological replicates. We found that all eight proteins exhibited significant increases in protein levels during oocyte maturation (Fig. $3 \mathrm{~J}, \mathrm{~K})$. Interestingly, we found that protein increases were modest at GVBD $+3 \mathrm{~h}$, which is the final time point that we used to analyze mRNA association with polysomes. However, we observed robust increases in protein levels at GVBD $+20 \mathrm{~h}$, suggesting that increased mRNA association with polysomes precedes increased protein production. In contrast, we also analyzed the protein levels of several proteins that showed decreased mRNA polysome association and were unable to detect a decrease in protein level for any proteins tested (data not shown). This suggests that these proteins may be stable during oogenesis, and decreases in mRNA association with polysomes do not lead to rapid changes in protein levels unless they are coupled with active proteolysis of the preexisting protein.

To determine if oocytes change the translated fraction of specific classes of transcripts we performed STEM clustering of mRNAs based on translation behavior. We identified two major (and four minor) clusters of mRNA translation behavior (Fig. 3G-I). The first cluster contains 722 transcripts ( $11 \%$ of measured) and exhibits a dramatic decrease in the fraction of mRNA present on polysomes (Fig. 3G). GO analysis demonstrates that ribosomal, cell-cell adhesion, and mitochondrial protein transcripts are highly enriched, similar to the GO categories enriched in deadenylated and degraded (Fig. 2) mRNAs (Supplemental Table 7). Cluster 2 contains 313 transcripts ( $\sim 5 \%$ of measured) and exhibits a significant, rapid increase in mRNA fraction present on polysomes (Fig. $3 \mathrm{H}$ ). $\mathrm{GO}$ analysis demonstrates that cell cycle/mitosis and splicing proteins are highly enriched in this cluster (Supplemental Table S8). Similar to polyadenylated transcripts, many proteins involved in kinetochore formation, sister chromatid cohesion, and cyclins that are required for completion of meiosis were up-regulated. We also identified proteins regulating DNA replication and sister chromatid cohesion that are likely not needed until the early embryonic divisions. We also identified a smaller cluster of 57 transcripts that slowly increases the fraction of mRNAs present on polysomes (Fig. 31; Supplemental Table S9). This cluster contains meiotic cyclins and Emi2, and Securin, which may be required to maintain the MII arrest after oocyte maturation. Taken together these results show that oocytes regulate mRNA translation as they mature and that specific functional classes of transcripts are up- or down-regulated. 
A
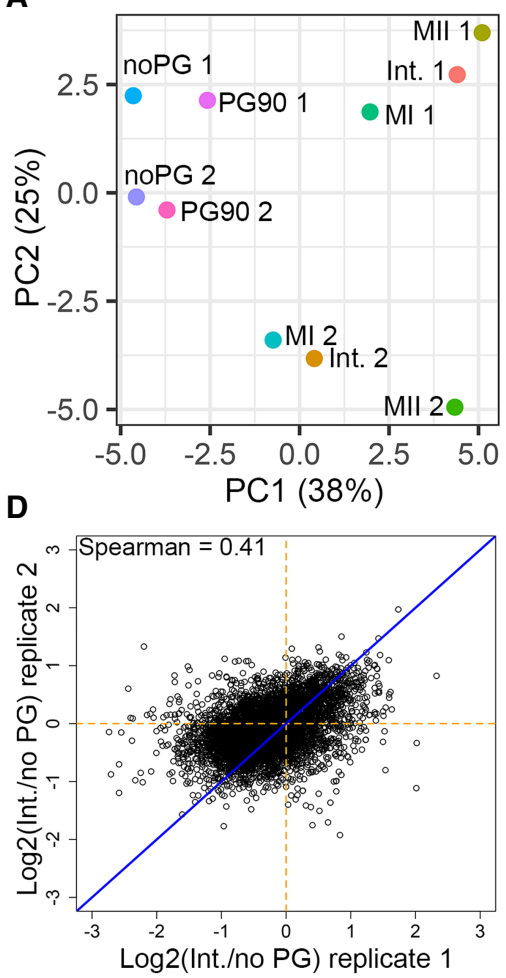

G
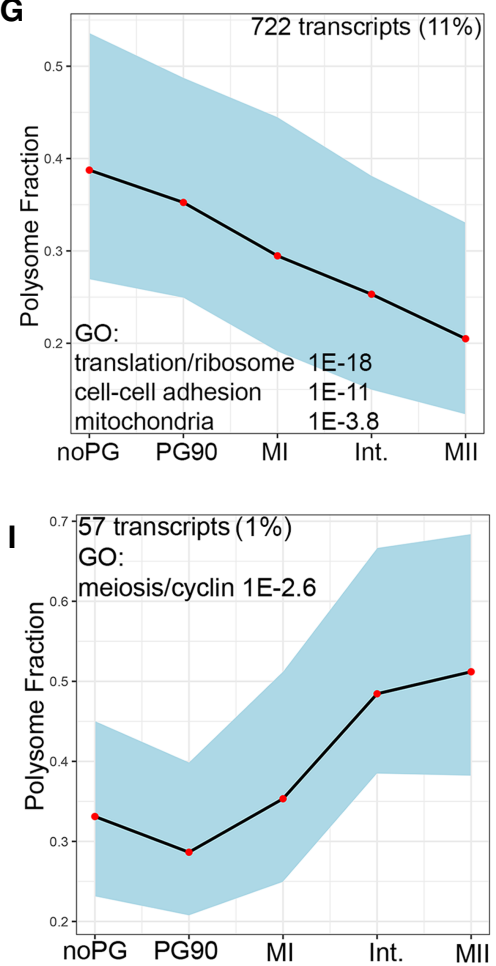

B

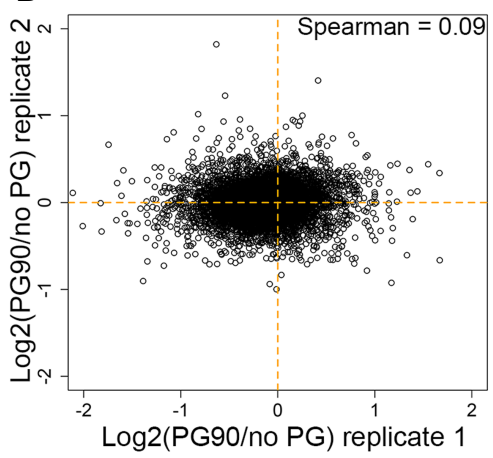

E

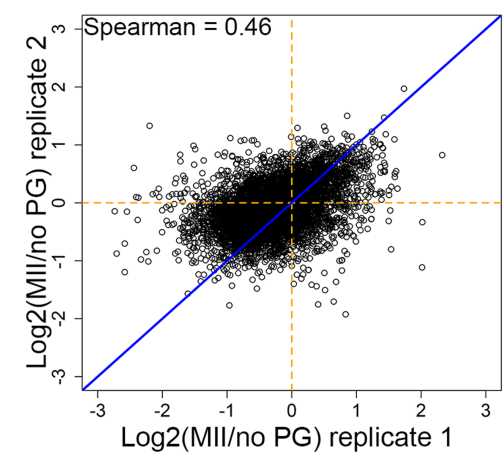

H

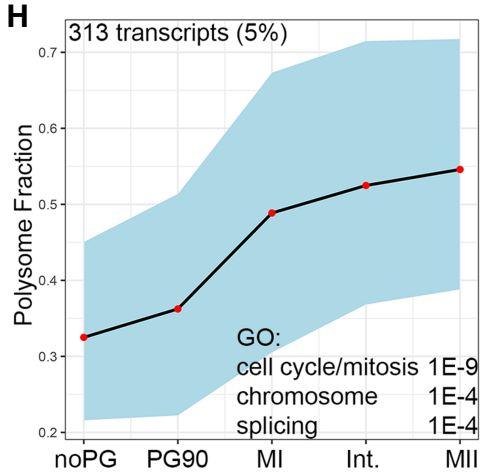

K 8

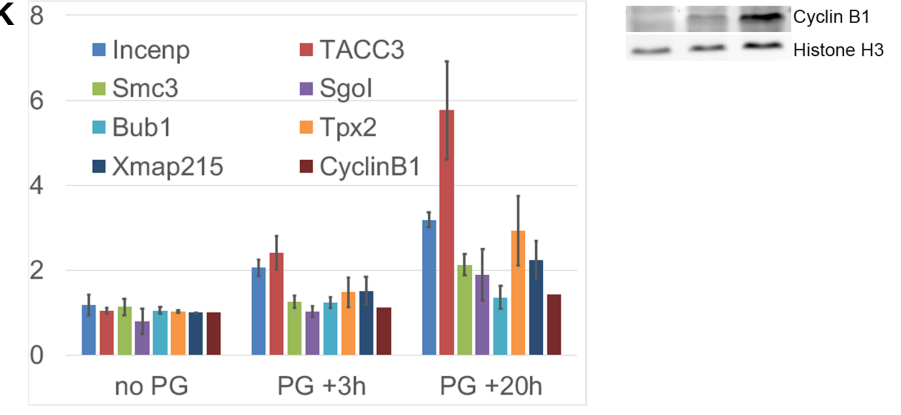

\section{C}

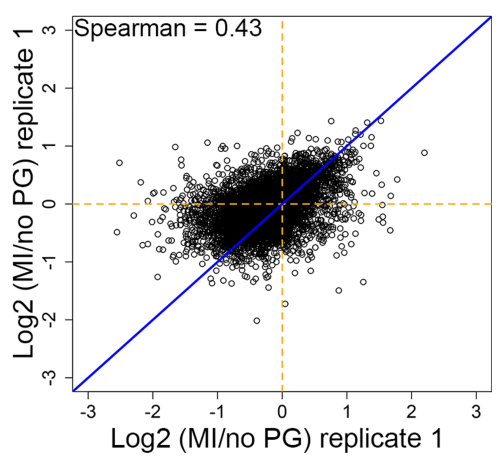

F

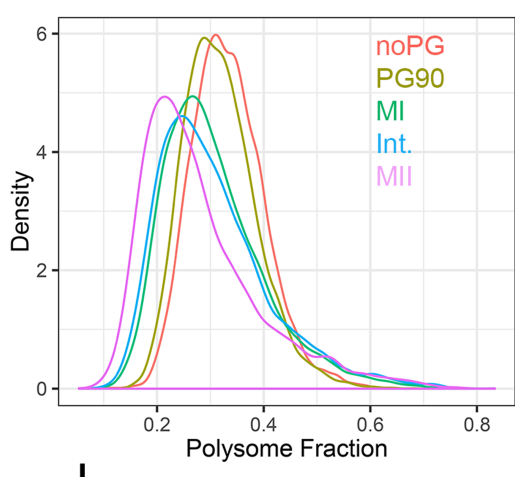

J

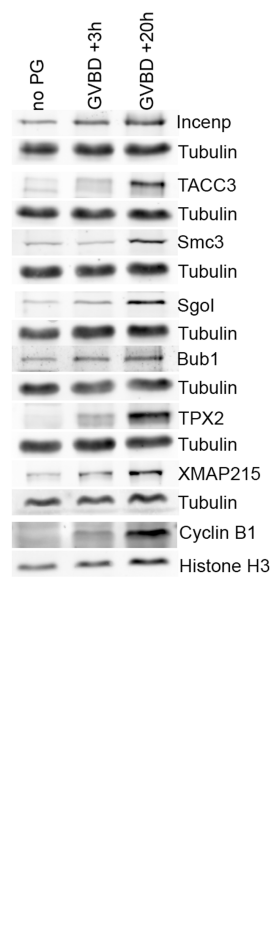

FIGURE 3. Translational changes during oocyte maturation. (A) Extracts from oocytes at different stages of maturation were separated on sucrose gradients and the fraction of mRNA associated with polysomes was calculated. PCA analysis and plot shows the relationship between different oocyte stages and biological replicates. (B-E) Scatterplots comparing changes in mRNA polysome fraction between replicate extracts. Spearman correlation coefficients are indicated on the plots. (F) Histogram plot showing the fraction of mRNA associated with polysomes at different stages of oocyte maturation. (G) STEM cluster of translationally repressed mRNAs and associated GO terms. (H) STEM cluster of translationally activated mRNAs and associated GO terms. (I) STEM cluster of slowly translationally activated mRNAs and associated GO terms. (J) Western blots of candidate proteins predicted to be translationally activated during oocyte maturation. (K) Quantitation of western blots from two biological replicates. 


\section{Complex relationship between polyadenylation and translation}

To understand the relationship between transcript polyadenylation and translation, we combined our Tail-seq and polysome data. We analyzed 2093 transcripts with reliable data for both poly(A) tail length and polysome fraction in both biological replicates (Supplemental Table 10). We analyzed the correlation between changes in poly(A) tail length and changes in the fraction of mRNA on polysomes across oocyte maturation (Fig. 4A-E). At the early stages of meiosis, changes in poly $(\mathrm{A})$ tail length and translation were very weakly correlated, but the correlation between the two factors improved as oocytes progressed through maturation (Fig. 4E). In general, increases in poly(A) tail length were positively correlated with an increased fraction of mRNA on polysomes and decreased poly(A) tail length positively correlated with a decreased mRNA polysome fraction. Interestingly, we observed a clear temporal offset in correlation between poly $(A)$ changes and translation changes (Fig. 4E), which is consistent with studies of mos and cyclin B1 during $X$. laevis oocyte maturation (de Moor and Richter 1997). This is especially well illustrated when comparing changes in poly(A) tail length in $\mathrm{MI}$ to changes in translation at MI, Int., and MII. The poly(A) tail changes are generally better correlated with polysome changes at a later developmental stage, suggesting that poly $(A)$ tail changes precede translational changes. Additionally, we examined the correlation between raw poly(A) tail length and fraction of mRNA present on polysomes (Fig. 4F). We observed that poly(A) length was also positively correlated with polysome fraction, suggesting that both raw poly(A) tail length and changes in poly $(A)$ tail length contribute to changes in mRNA translation.

To determine how changes in polyadenylation affect translation for a defined set of transcripts, we examined the translational behavior of clusters of mRNAs exhibiting increased or decreased polyadenylation (Fig. 1K,L) during oogenesis. We used STEM software to cluster transcripts with decreased poly $(A)$ tail length based on translational behavior (Fig. 4G). We found that all deadenylated transcripts had reduced mRNA levels on polysomes (Fig. 4G). In contrast, when we clustered polyadenylated transcripts based on translational behavior, we found two distinct groups of translational behavior (Fig. $4 \mathrm{H}, \mathrm{I}$ ). First, 58\% of transcripts showed both increased poly(A) tail lengths and increased translation, consistent with a positive correlation between longer poly $(A)$ tails and increased translation. Surprisingly, we also identified a second cluster (42\% of transcripts) that showed decreased polysome association despite having increased poly(A) tails. Interestingly, polyadenylated mRNAs with down-regulated translation exhibited significantly shorter poly(A) tails than translationally activated mRNAs, suggesting that there may be a poly (A) tail length threshold necessary to strongly activate translation (Fig. 4H-J). Decreased translation of some polyadenylated mRNA could be influenced by the number of PAB1 binding sites in the poly(A) tail (Eliseeva et al. 2013), by the presence of specific sequence elements in the $3^{\prime}$ UTR, or $3^{\prime}$ UTR length (see Fig. 6). Taken together, our results indicate that mRNA deadenylation always leads to decreased polysome association of mRNAs. In contrast, mRNA polyadenylation can lead to both unchanged and increased translation, suggesting that additional mRNA features may control translation in addition to mRNA poly $(A)$ tail length.

\section{Genome-wide identification of polyadenylation sites and prediction of $3^{\prime}$ UTR sequences}

In order to understand if sequence elements located in $3^{\prime}$ UTRs control the polyadenylation and translation behavior of mRNAs, we undertook an analysis of mRNA $3^{\prime} U T R$ s. Many genes have multiple possible polyadenylation sites (PASs) whose use can differ based on developmental context (Ulitsky et al. 2012; Hu et al. 2017; Tian and Manley 2017; Zhou et al. 2019). In order to accurately annotate PASs in X. laevis oocytes, we performed PAS-seq (Wang et al. 2017) in Stage VI oocytes. After read filtering and peak calling we identified PASs supported by $\geq 4$ independent reads (Supplemental Table 11). The majority of PASs mapped to $3^{\prime}$ UTRs (Fig. 5A). Interestingly, PAS-seq identified unannotated $3^{\prime}$ UTR regions (Fig. 5D) and improved our ability to analyze oocyte transcript $3^{\prime} U T R$ regions. PAS sites mapped to 12,888 genes with the majority of transcripts having a single PAS site (Fig. 5B,D,E). However, many transcripts clearly contained multiple PAS sites (Fig. 5F) that are used in differing ratios. For all subsequent analysis, we calculated PAS usage by counting the number of reads supporting each PAS and only considering the $3^{\prime}$ UTRs corresponding to PASs with the greatest number of reads.

To explore the general characteristics of PASs, we searched for the presence of a canonical polyadenylation signal [poly(A) signal] (AAUAAA or AUUAAA) and found that $85 \%$ contained at least one poly(A) signal. Additional$l y$, we determined the distance from the most 3 ' polyadenylation signal to the measured $3^{\prime}$ end of the transcript. As observed with human RNAs (Wu and Bartel 2017) we found that most RNAs contained a polyadenylation signal $\sim 24 \mathrm{nt}$ from the $3^{\prime}$ end of the RNA (Fig. 5C). Collectively, PAS-seq allowed us to experimentally identify $3^{\prime}$ ends of transcripts in $X$. laevis oocytes and to accurately predict 3'UTR sequences for analysis.

\section{3'UTR length and primary sequence motifs control transcript polyadenylation and translation}

To determine what transcript features influence polyadenylation and translation in oocytes, we analyzed the length and composition of motifs in experimentally determined 
A

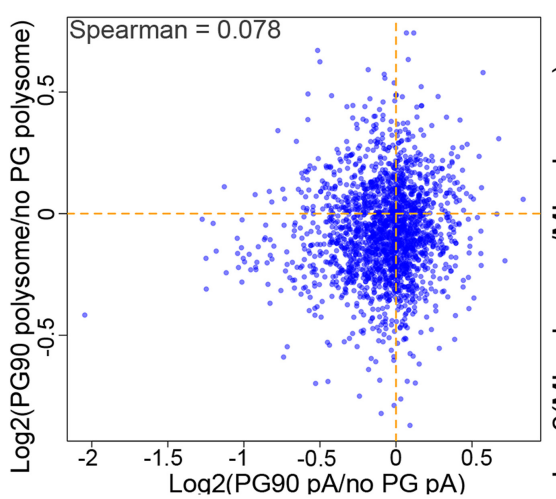

D

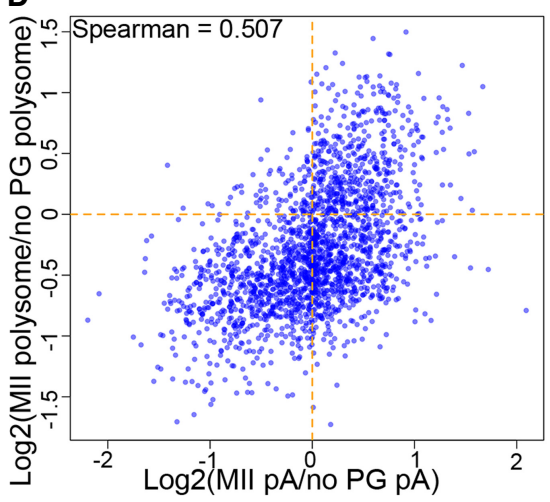

G

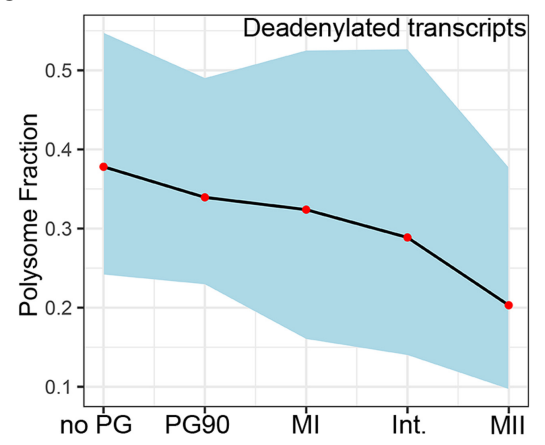

B

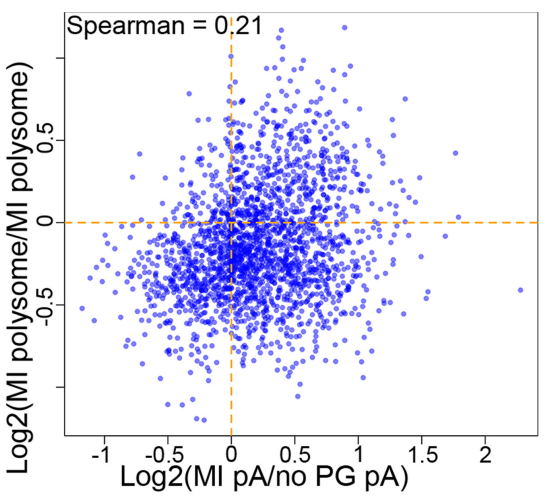

E

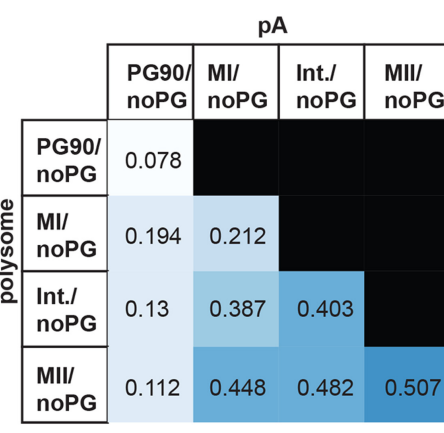

C

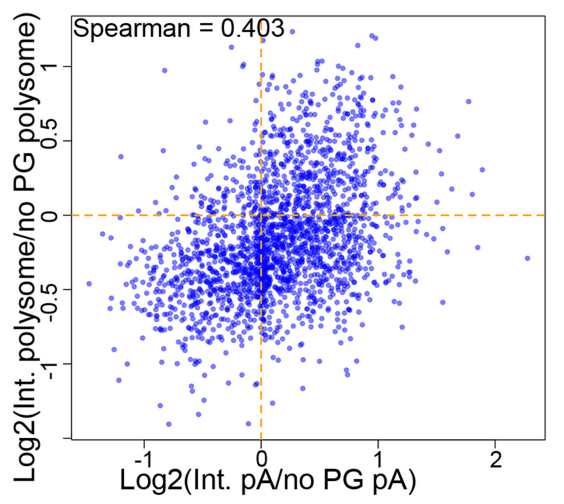

F

0-0.1

$0.1-0.2$

0.2-0.3

0.3-0.4

0.4-0.5

0.5-0.6

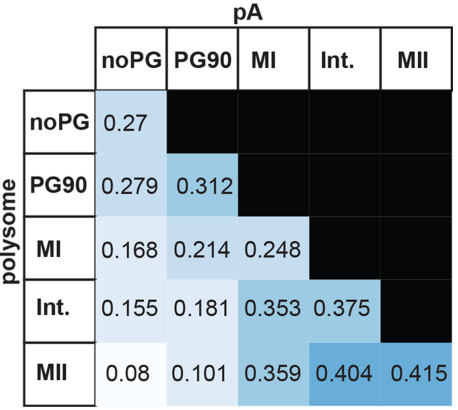

H

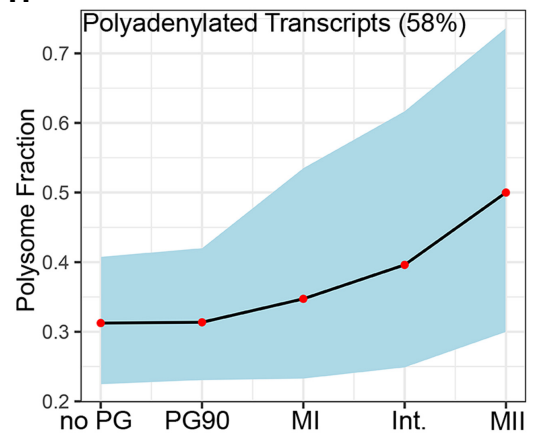

I

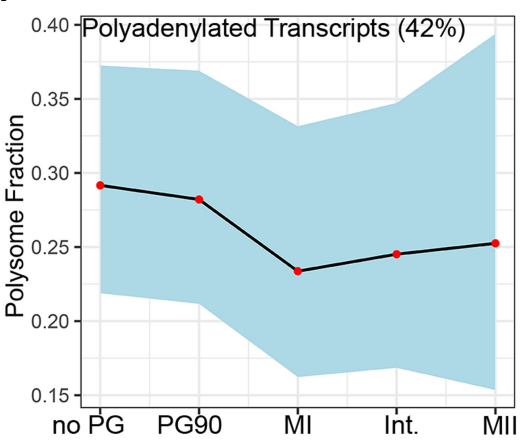

J

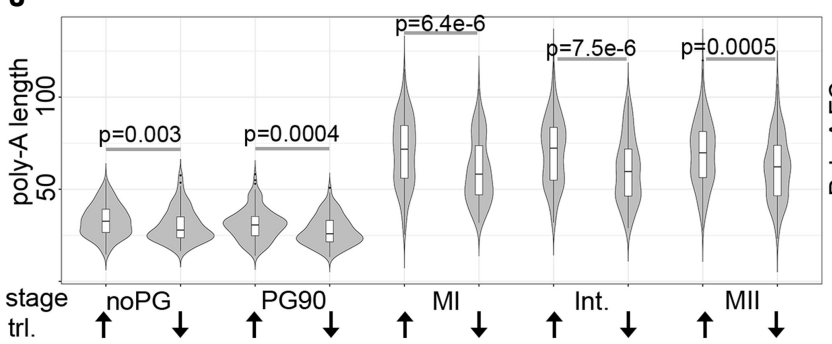

K

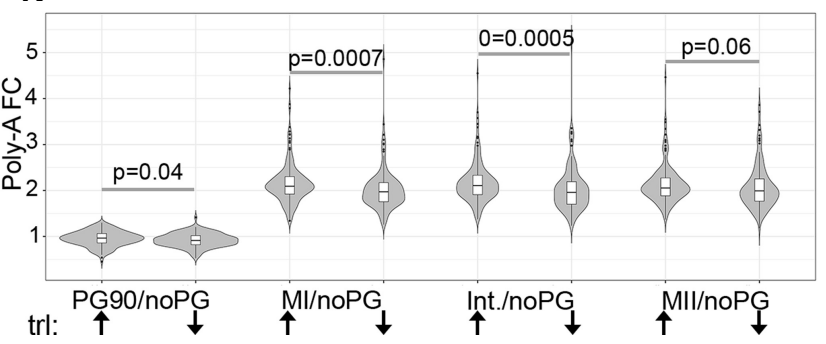

FIGURE 4. Correlation between transcript polyadenylation and translation. (A-D) Scatterplots of the change in polyadenylation compared to the change of the fraction of mRNA present on polysomes. (E) Spearman correlation coefficients between changes in polyadenylation and changes in mRNA fraction on polysomes. $(F)$ Spearman correlation coefficients of raw poly $(A)$ tail length and raw polysome percentage measurements. $(G)$ The translation behavior of deadenylated transcripts (from Fig. 1H) was examined using STEM software. All deadenylated transcripts exhibited translational repression. $(H, I)$ The translation behavior of adenylated transcripts (From Fig. 1G) was examined using STEM software. Adenylated transcripts were either translationally activated $(H)$ or repressed $(I)$. $(J)$ Violin plots of the raw poly $(A)$ tail lengths of polyadenylated transcripts that were translationally activated (up arrow) or repressed (down arrow). (K) Violin plots of the fold change in poly(A) tail length of adenylated transcripts that were translationally activated (up arrow) or repressed (down arrow). Indicated $P$-values are the result of a Wilcoxon rank-sum test. 
A 3'UTR $(15,805)$

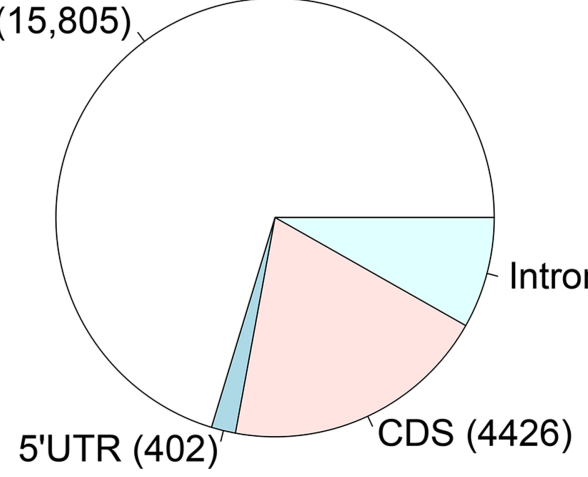

C

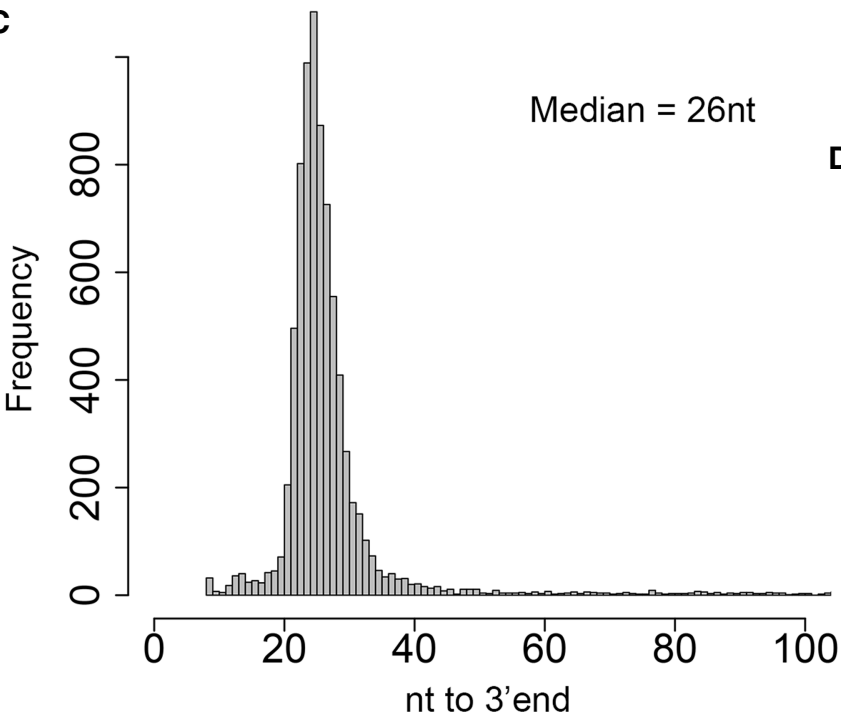

E

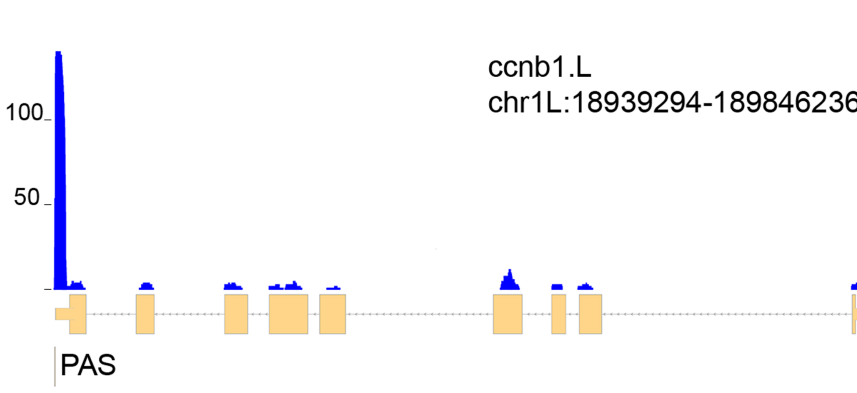

(1844)
D

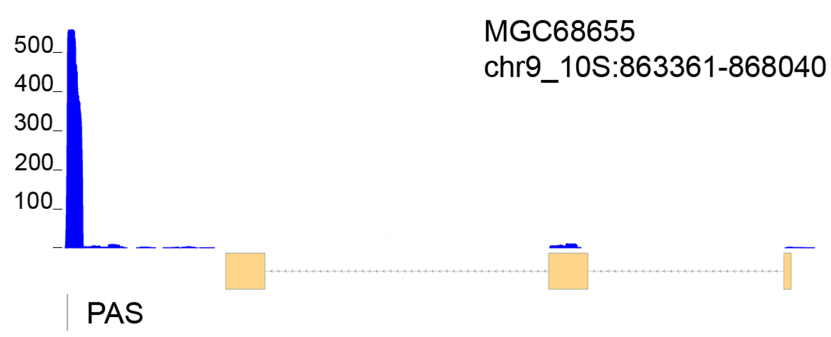

$\mathbf{F}$

30 mos.L

chr6L:116460751-11646604؛

FIGURE 5. PAS-seq analysis of the $X$. laevis oocyte transcriptome. (A) PAS-seq was used to identify PASs in $X$. laevis oocytes. Pie chart shows the fraction of PASs mapping to each type of transcript feature. (B) Histogram of the number of PASs per gene. (C) Histogram of the average number of nucleotides from the $3^{\prime}$ most base of the polyadenylation signal (AAUAAA, AUUAAA) to the measured end of the transcript. (D,E) Genome browser views of PAS-seq reads compared to three genes. PAS calls are listed below each gene as thin bars.

3'UTR sequences. mRNA 3'UTR length can influence translation efficiency and stability, with shorter 3'UTRs having a tendency toward higher stability and translation (for review, see Gruber and Zavolan 2019). To study if the length of $3^{\prime}$ UTRs can modulate translation of polyadenylated mRNAs, we examined $3^{\prime} U T R$ length in clusters of transcripts with differential association with polysomes. We found that transcripts with increased polyadenylation but decreased translation exhibited significantly longer 3'UTR sequences compared to mRNAs that were adenylated and translationally activated (Fig. 6A). Longer $3^{\prime} U T R s$ are likely to contain increased numbers of regulatory elements that could decrease translation. Interestingly, deadenylated and translationally repressed transcripts 
A

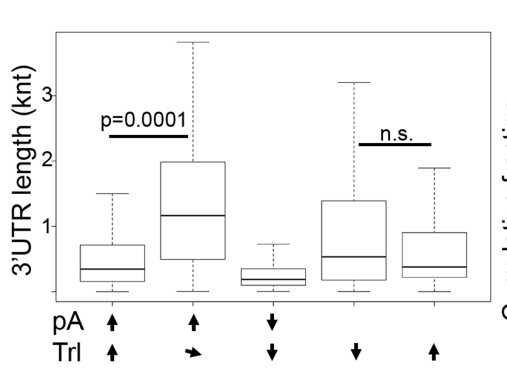

D

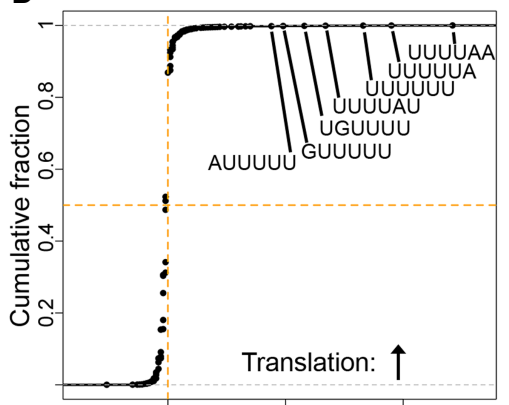

Enriched motif density -0.5 control motif density

G

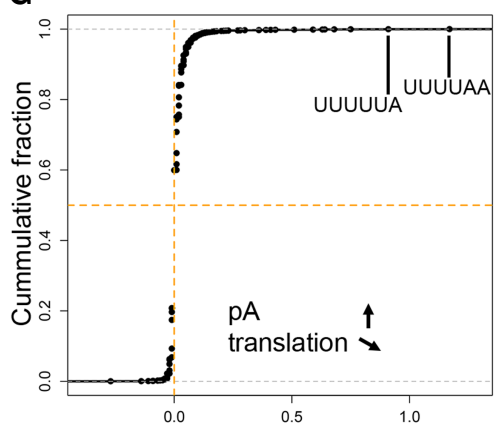

Enriched motif density - control motif density

\section{J}

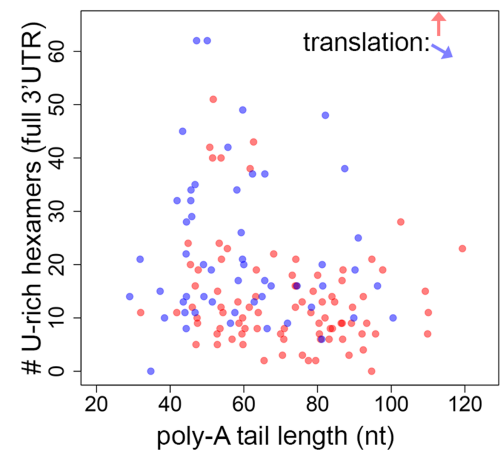

B

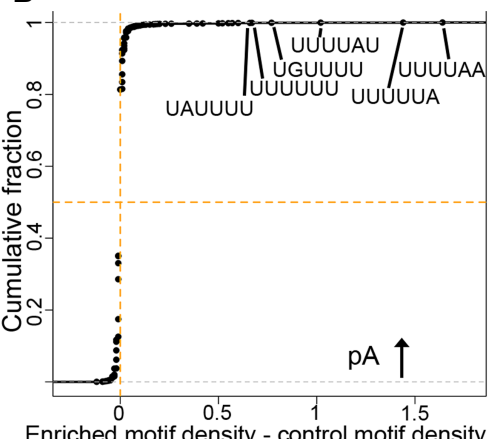

Enriched motif density - control motif density

E

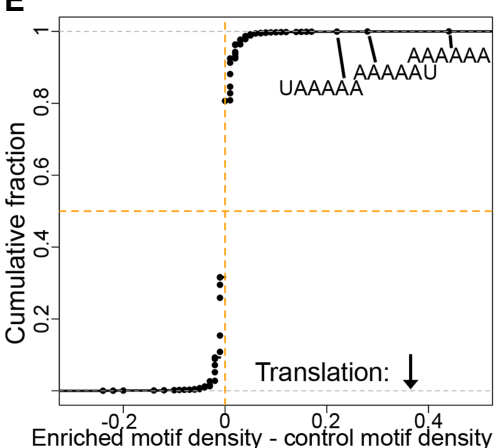

H。

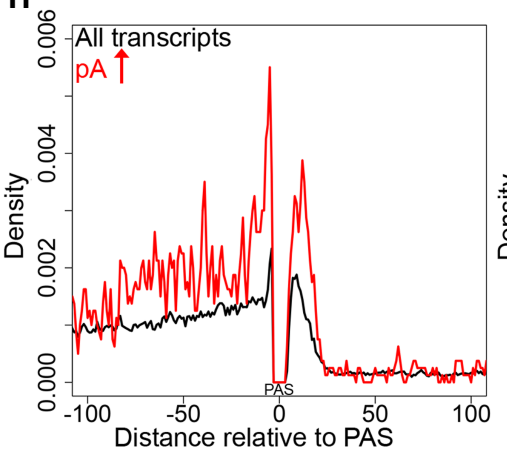

K

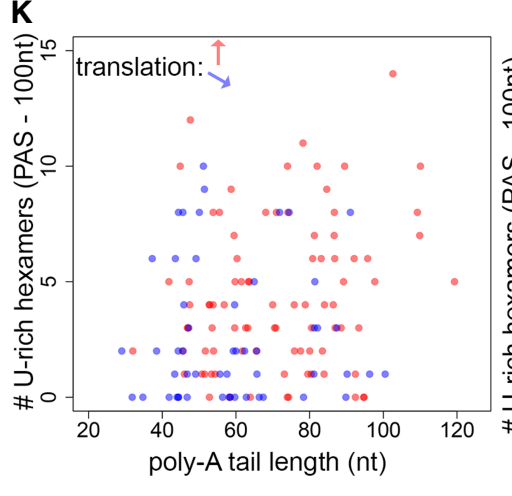

C

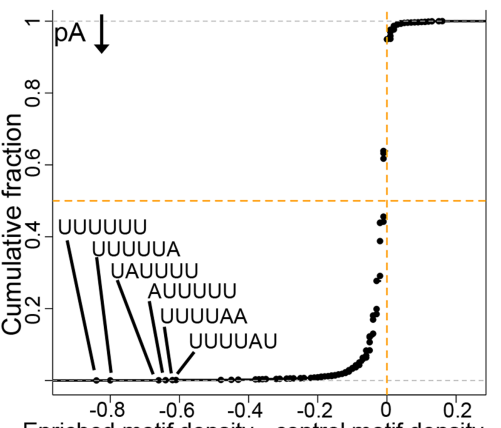

Enriched motif density - control motif density

F

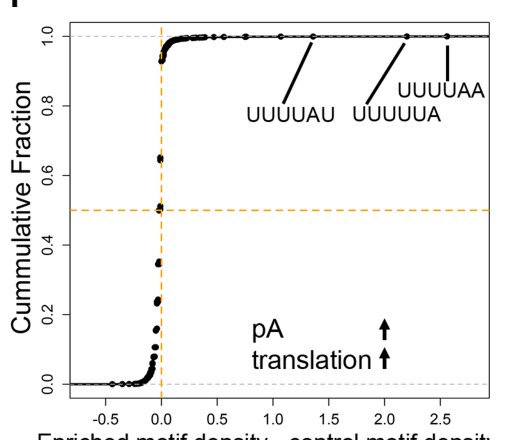

Enriched motif density - control motif densit)

I

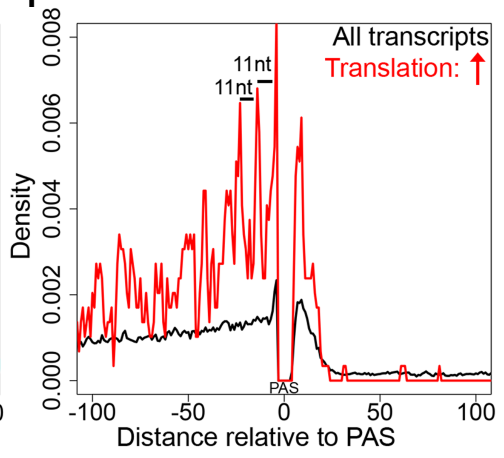

L U-rich hexamer density near PAS

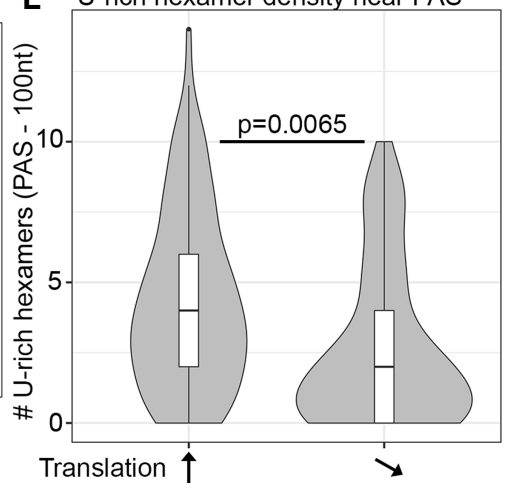

FIGURE 6. Primary sequence motifs are enriched in polyadenylated and translationally activated transcripts. (A) $3^{\prime} U T R$ s lengths were measured from clusters of $m$ RNAs exhibiting various polyadenylation and translation behavior. $P$-values are the result of a Wilcoxon rank sum test. (B-E) The density of all hexamers was calculated in the $3^{\prime}$ UTRs of various sets of transcripts (polyadenylated [B], deadenylated [C], translationally activated $[D]$, translationally repressed $[E]$ ) and in all transcripts. Cumulative distribution plots show the enrichment of various hexamers in different subsets of transcripts compared to all transcripts. $(F, G)$ Hexamer enrichment was calculated in the $3^{\prime} U T R$ s of polyadenylated transcripts that were translationally activated $(F)$ or translationally repressed $(G)$. Translationally activated transcripts exhibit a higher density of $U$-rich sequence elements. $(H)$ The positions of the top $13 \mathrm{U}$-rich elements (from B) were compared to the 3'-most polyadenylation signal for all transcripts (black line) and adenylated transcripts. (I) The relative density of U-rich sequences compared to the $3^{\prime}$-most polyadenylation signal for translationally activated transcripts. $(J)$ Scatterplot of poly $(A)$ tail length (at MII) and the number of U-rich hexamers in the entire $3^{\prime} U T R$ for translationally activated (red) or repressed (blue) mRNAs. (K) Same plot as in J except that U-rich element density is only compared in the $100 \mathrm{nt} 5^{\prime}$ of the PAS sequence. (L) Violin plot of U-rich element density in the $100 \mathrm{nt} 5^{\prime}$ of the PAS sequence in translationally activated and repressed mRNAs. $P$-value is the result of a Wilcoxon rank-sum test. 
did not exhibit longer 3'UTR sequences. Additionally, translationally activated and repressed transcripts (Figs. 3C,D, 6A) did not exhibit significant differences in $3^{\prime}$ UTR length, suggesting that multiple mechanisms control mRNA translation.

To determine if specific sequence elements are enriched in clusters of transcripts with coordinated behavior, we calculated the enrichment of all hexamers (Lambert et al. 2015) (as a density of motifs per knt) in clusters of transcripts compared to all transcripts. We first compared the groups of transcripts that were polyadenylated or deadenylated during oocyte maturation. A large family of $U$-rich hexamers were enriched in polyadenylated transcripts (Fig. 6B; Supplemental Table S12). The U-rich motifs are similar to and include the CPE element (UUUUAA or UUUAAU) which is well documented to promote transcript polyadenylation, suggesting that these motifs are likely binding sites for CPEB1 (Mendez and Richter 2001; Afroz et al. 2014; Dominguez et al. 2018). In contrast, deadenylated transcripts did not contain any enriched hexamers (Fig. 6C; Supplemental Table S13), but exhibited a significant depletion of the family of U-rich hexamers. Collectively, these data suggest a model for how cis-acting sequence elements control transcript polyadenylation. A high density (or specific location, see below) of U-rich sequence elements promotes transcript polyadenylation while an absence of $\mathrm{U}$-rich elements promotes transcript deadenylation. The data suggest that transcript deadenylation is the default behavior during oocyte maturation and that deadenylation is counteracted by the presence of U-rich sequences in the $3^{\prime}$ UTR.

To determine if cis-acting sequence elements also influence the translation behavior of mRNAs, we calculated hexamer enrichment in translationally up- and downregulated mRNAs. Similar to polyadenylated transcripts, a large family of U-rich hexamers were also enriched in translationally activated mRNAs (Fig. 6D; Supplemental Table S14), with the CPE element being the most enriched element. In contrast, only a few A-rich motifs were very modestly enriched in translationally repressed mRNAs (Fig. 6E; Supplemental Table S15). To determine if the density of $\mathrm{U}$-rich elements affects the translation behavior of polyadenylated mRNAs, we compared the density of $U$ rich hexamers in mRNAs that are polyadenylated and translationally activated (Figs. 4H, 6F) or translationally repressed (Figs. 4I, 6G). Interestingly, we found that the density of U-rich elements is much higher in translationally activated mRNAs compared to translationally repressed mRNAs (Fig. 6F,G). To understand how the location and density of U-rich elements influence polyadenylation and translation, we examined motifs in translationally activated (Fig. 6F) and repressed (Fig. 6G) mRNAs. We found that translationally repressed mRNAs had shorter poly(A) tails (Figs. 4J, 6J) and a higher raw number of $U$-rich hexamers across the entire $3^{\prime} U T R$ than translationally activated
mRNAs (Fig. 6J), which can be attributed to longer 3'UTR sequence (Fig. 6A). In contrast, U-rich element density in the $100 \mathrm{nt} 5^{\prime}$ of the PAS site is significantly higher in translationally activated mRNAs compared to translationally repressed mRNAs (Fig. 6K,L). Collectively, these results suggest that a high density of U-rich sequence elements promote both polyadenylation and polyadenylation-dependent translational activation, and a low density of $\mathrm{U}$-rich elements promote polyadenylation but not translation activation. These results are consistent with the strong correlation between transcript polyadenylation and translation (Fig. 4) and the known role of the CPE element in promoting polyadenylation and translation. Additionally, the identification of simple sequence motifs is consistent with a recent analysis of the preferred binding motifs of a large number of RNA-binding proteins (Ray et al. 2013; Dominguez et al. 2018).

Extensive analysis of the role of CPE elements in the regulation of polyadenylation and translation has demonstrated that the location of the CPE relative to the polyadenylation signal and to other CPE elements has a strong influence on CPE functionality (Piqué et al. 2008; Weill et al. 2017). To determine if U-rich sequence elements are located at specific positions in groups of mRNAs with coordinated behaviors, we analyzed the location of U-rich hexamers relative to the poly(A) signal. U-rich sequences were modestly enriched immediately before and after the polyadenylation signal in all transcripts (Fig. $6 \mathrm{H}, \mathrm{l}$, black line), but showed no enrichment at other positions. In contrast, polyadenylated transcripts exhibited strong peaks of U-rich elements before and after the polyadenylation signal as well as multiple secondary peaks $5^{\prime}$ of the poly(A) signal (Fig. 6H). Translationally activated transcripts also exhibited a strong enrichment of U-rich elements before and after the polyadenylation signal (Fig. 61). Additionally, translationally activated mRNAs exhibited several phased peaks $5^{\prime}$ of the polyadenylation signal that are separated by $\sim 11 \mathrm{nt}$. The appearance of phased U-rich elements fits well with the observation that CPE sites separated by 10-12 nt lead to translational repression of cyclin mRNAs before oocyte maturation, and that the distance between the CPE and poly(A) signal regulates polyadenylation-dependent translational activation (Piqué et al. 2008). Collectively, our data suggest that U-rich sequence elements are enriched near the polyadenylation signal of mRNAs that are polyadenylated and translated during oocyte maturation. Additionally, upstream U-rich element density, spacing, and $3^{\prime} U T R$ length likely contribute to polyadenylation and translational activation (Fig. 8).

\section{U-rich elements control translation of the Spc25.L mRNA during oocyte maturation}

Our results identified a family of U-rich sequence elements enriched in the $3^{\prime}$ UTRs of transcripts that are 
polyadenylated and translated during oocyte maturation. To test the importance of U-rich sequence elements in protein translation during oogenesis, we used luciferase assays to examine the effects on sequence elements in the $3^{\prime}$ UTR of the Spc25.L 3'UTR. We appended WT or mutant Spc25.L 3'UTR sequences to Firefly luciferase, injected the mRNAs into oocytes, and measured basal translation and translation activation after oocyte activation. The Spc25.L 3'UTR contains five U-rich hexamers, four $5^{\prime}$ of the PAS, and one $3^{\prime}$ of the PAS (Fig. 7A). The Spc25.L 3'UTR fused to Firefly luciferase led to strong translational activation during oocyte maturation (Fig. 7C). Mutation of the U-rich element $3^{\prime}$ of the PAS led to lower basal translation in unactivated oocytes (Fig. 7B), but did not affect translational activation during oocyte maturation. Mutation of any three U-rich elements located $5^{\prime}$ of the PAS resulted in decreased translational activation during oocyte maturation (Fig. 7C). Collectively, these results support the hypothesis that a high density of U-rich hexamers located near the PAS regulate translation activation during oocyte maturation.

\section{DISCUSSION}

We used a combination of genome-wide approaches to provide a high temporal resolution view of transcript polyadenylation and translation during vertebrate oocyte maturation. We found large-scale changes in both poly $(A)$ tail lengths and translation, suggesting that oocytes execute a precise post-transcriptional gene expression program to complete the meiotic divisions and prepare for early development following fertilization. Our work identified cis-acting sequence elements whose density and location may set a threshold for activation of polyadenylation-dependent translation and is likely a rich source of novel proteins that facilitate oocyte maturation (Fig. 8).

\section{Large-scale changes in poly(A) tail length during oocyte maturation}

Several recent genome-wide studies demonstrated that large-scale changes in transcript polyadenylation occur during oogenesis and early development (Chang et al. 2014; Subtelny et al. 2014; Eichhorn et al. 2016; Lim et al. 2016; Park et al. 2016). In particular, two recent studies found large changes to poly(A) tail length and a strong correlation between changes to poly(A) tail length and changes in translation in Drosophila oocytes (Eichhorn et al. 2016; Lim et al. 2016). However, it is not possible to experimentally control the progress of oocytes through maturation in Drosophila and it was not clear how changes in $\operatorname{poly}(A)$ tail length are related to the morphological events of oocyte maturation. In addition, Drosophila oocytes arrest in metaphase of meiosis I prior to fertilization while vertebrate oocytes arrest at metaphase of meiosis II, so it is not clear if Drosophila is an accurate model of vertebrate oocyte maturation. We confirmed that a large fraction of the transcriptome changes poly(A) tail length during oocyte maturation. We found that most of the transcriptome is polyadenylated as oocytes undergo GVBD and that the majority of transcripts are then deadenylated as oocytes progress through the meiotic divisions. Global transcript polyadenylation is similar to the global polyadenylation observed when comparing mature and immature Drosophila oocytes (Eichhorn et al. 2016). Interestingly, we found that a subset of transcripts that code for proteins associated with mitosis retain long poly(A) tails through oocyte maturation. Based on the functions of these proteins, it is likely that polyadenylation facilitates production of proteins necessary for the completion of meiosis as well as proteins that will not be needed until the mitotic divisions of early development. As a result of the experimental tractability of $X$. laevis oocytes we were able to obtain a high temporal resolution view of poly $(A)$ and translation changes during oocyte maturation. We found that changes in $\operatorname{poly}(A)$ tail length precede changes in translation. Collectively, our work adds to the growing body of evidence that global poly(A) tail length changes are a highly conserved feature of oocyte maturation and are used to control gene expression in transcriptionally silent cells.

Pioneering work in $X$. laevis demonstrated that cis-acting sequence elements, such as the CPE, control cytoplasmic polyadenylation in Xenopus oocytes (Sheets et al. 1994;
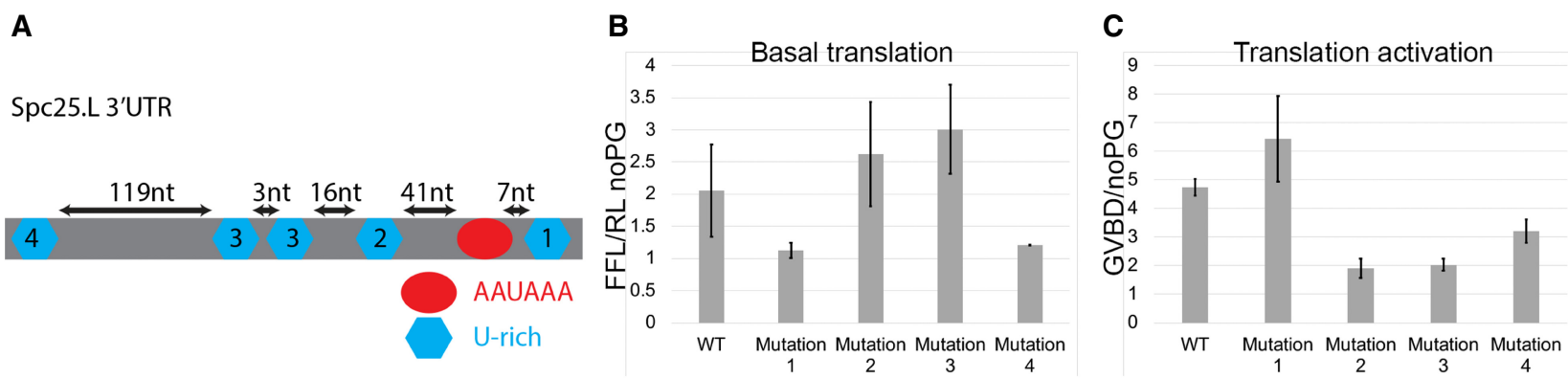

FIGURE 7. (A) Schematic diagram of Spc25.L 3'UTR with positions of U-rich hexamers and PAS indicated. (B) Normalized translation activity at noPG of Firefly luciferase fused to WT or mutant Spc25.L 3'UTR sequences. (C) Relative translation activation GVBD/noPG for WT and mutant Spc25.L 3'UTR sequences. 


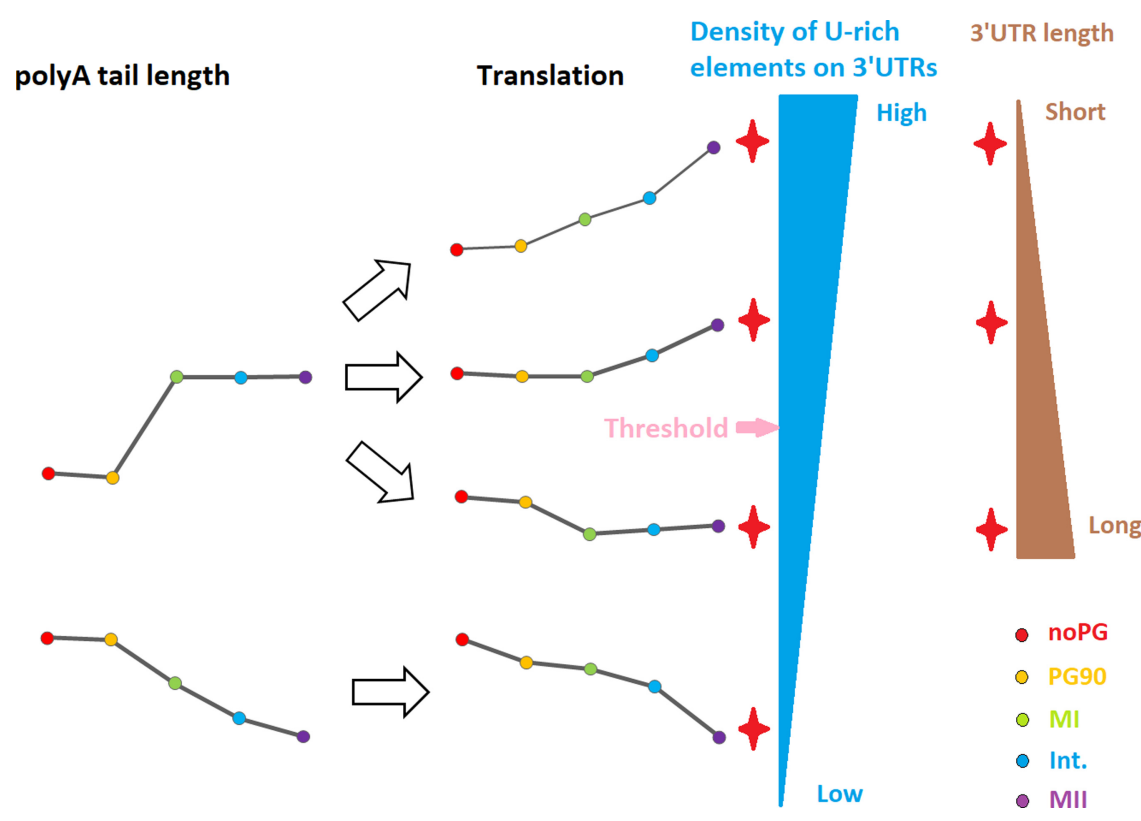

FIGURE 8. Model for control of polyadenylation and translation by U-rich sequence elements and $3^{\prime}$ UTR length.

Stebbins-Boaz et al. 1996). In-depth analysis of the mos and cyclin B1 mRNAs demonstrated that cytoplasmic polyadenylation is strongly influenced by the number and positions of the CPE element relative to the polyadenylation signal (Piqué et al. 2008; Weill et al. 2017). However, it has not been possible to determine if there is a genomewide correlation of the CPE element with cytoplasmic polyadenylation or to determine how the CPE positions globally influence cytoplasmic polyadenylation. To address this issue, we used PAS-seq to identify the PASs of transcripts in immature oocytes and experimentally defined 3'UTR to search for motifs enriched in polyadenylated and deadenylated transcripts. Interestingly, we found a large family of U-rich sequence motifs that were enriched both before and after the polyadenylation signal in polyadenylated transcripts. Additionally, we could detect secondary enrichment peaks for U-rich hexamers, consistent with previous work suggesting that clusters of precisely spaced CPE elements promote efficient cytoplasmic polyadenylation (Piqué et al. 2008). The fact that we identified many different U-rich motifs, including the $C P E$, suggests that CPEB proteins likely can bind to a range of $U$-rich sequences, which is consistent with recent work (Afroz et al. 2014; Dominguez et al. 2018). Surprisingly, we found that no sequence motifs were enriched in deadenylated transcripts, but rather that deadenylated transcripts were characterized by an absence of U-rich motifs. Interestingly, recent work in Zebrafish also identified a family of U-rich sequence elements that promote mRNA stability during early development (Vejnar et al. 2019). Taken together, these results suggests that transcript deadenylation is the default behavior as oocytes mature and that deadenylation is der to prepare the oocyte for early developmental divisions following fertilization (Vastenhouw et al. 2019). Following fertilization maternal transcripts are degraded as embryos transition from maternal to zygotic control of gene expression. Global transcript deadenylation is known to be activated in mature oocytes and early embryos (Voeltz and Steitz 1998) and could be the beginning of clearance of maternal transcripts prior to zygotic genome activation (Vastenhouw et al. 2019).

\section{Translation changes during oocyte maturation}

To determine if poly $(A)$ tail length and translation are correlated during vertebrate oocyte maturation, we measured the fraction of each mRNA associated with polyribosomes. We found that translation was globally repressed as oocytes progressed through the meiotic divisions, consistent with long-standing observations from somatic cells that protein translation is globally repressed during $\mathrm{M}$ phase (Prescott and Bender 1962; Pyronnet et al. 2001; Tanenbaum et al. 2015). However, we identified a large group of transcripts that increased translation during oocyte maturation, including a group of transcripts coding for proteins involved in the completion of meiosis and proteins that are likely required for the early embryonic mitotic divisions. A large number of transcripts coding for proteins involved in mRNA splicing are also translationally activated, which is surprising because oocytes are transcriptionally silent (Ziegler and Masui 1976). It is tempting to speculate that splicing components are up-regulated in preparation for the onset of zygotic transcription.

Extensive work studying the CPE element has shown properly spaced CPE elements serve to translationally 
repress mRNAs prior to oocyte maturation and promote "early" (prophase), "late" (metaphase I), or "late late" translational activation following oocyte activation (Piqué et al. 2008). We found that a family of U-rich elements were enriched immediately before and after the polyadenylation signal in translationally activated mRNAs, which is predicted to activate "early" and "late" translational activation (Piqué et al. 2008). Additionally, we found strong, regularly spaced peaks of U-rich sequence elements at a spacing of $\sim 11 \mathrm{nt}$, which is strikingly similar to the optimal spacing of CPE elements found in cyclin mRNAs to promote translational repression prior to maturation and translational activation during maturation. Our results suggest that the same family of U-rich sequence elements is the major global mechanism that controls both mRNA polyadenylation and translation during oocyte maturation. These results are consistent with a family of U-rich elements that positively regulate mRNA translation during mouse oocyte maturation (Chen et al. 2011).

\section{Coordinated regulation of polyadenylation and translation during oocyte maturation}

During oogenesis and early development, poly(A) tail length and mRNA translation are well correlated in Drosophila, Xenopus, and zebrafish. Our results are consistent with previous studies in that we find that poly $(A)$ tail length and changes to poly(A) tail length are well correlated with changes in mRNA translation during vertebrate oocyte maturation. Interestingly, we find that poly(A) tail length is uncorrelated with translation in prophase-arrested oocytes and becomes more correlated as oocytes progress through meiosis. We found that changes in poly(A) tail length were better correlated with changes in translation than raw poly $(A)$ tail length. Importantly, our high temporal resolution data set allowed us to observe a temporal offset in changes in poly(A) tail length and changes in translation. This suggests that lengthening or shortening poly $(A)$ tails causes changes in translation initiation.

Examination of the translational behavior of polyadenylated and deadenylated groups of transcripts revealed important information about the relationship between the two processes. First, deadenylated transcripts were always translationally repressed, suggesting that transcript deadenylation and translational repression are the default regulation as oocytes progress through meiosis. Second, adenylated transcripts exhibit complex translational behavior. Consistent with work on the cyclin B and mos mRNAs, a majority of polyadenylated transcripts are translationally activated. However, a substantial fraction of polyadenylated transcripts show no change in translation or are translationally repressed. The major difference between translationally activated and unchanged transcripts is the density of upsteam U-rich elements near the polyadenylation signal. This novel result supports the hypothe- sis that the density and spacing of U-rich elements are the critical determinants for controlling translation. Previous work demonstrated that polyadenylation is always correlated with translational activation, but our work shows that a high density of U-rich elements is necessary to activated translation on polyadenylated transcripts. Low density of non-optimally spaced U-rich elements can promote polyadenylation, but the correct spacing is critical to promote translational activation. Comparison of our U-rich hexamers to high-throughput identification of RNA-binding protein motifs (Ray et al. 2013; Dominguez et al. 2018; Vejnar et al. 2019) suggests several candidate proteins that could bind to these elements: CPEB, hnRNPC, Fubp1, Tia1, Raly, Elav. Interestingly, some of these proteins are strongly associated with translational repression of mRNA (Zhang et al. 2016; Diaz-Munoz et al. 2017). The requirement for a high density of U-rich sequences to promote translation is consistent with the observation that the mRNAbinding protein Dazl, which recognizes U-rich sequences, is important for mRNA translation in mouse oocytes (Chen et al. 2011). Additionally, binding of increasing numbers of Dazl molecules to a mRNA promotes translation in Xenopus oocytes (Collier et al. 2005), suggesting that Dazl density may control mRNA translation at a genome-wide scale during oocyte maturation. Further work will be required to determine whether other RBPs with a preference for U-rich elements also regulate translation during oocyte maturation.

In summary, we have found that large-scale changes in protein expression occur during vertebrate oocyte maturation and that these changes are strongly coupled to changes in $\operatorname{poly}(A)$ tail lengths. Our work confirms work in other systems and suggests that cytoplasmic polyadenylation is the major mechanism regulating protein expression in transcriptionally silent cells. In addition, our work has identified a large group of transcripts that are translationally activated during oocyte maturation that could be a rich source of novel proteins important for the completion of oocyte meiosis.

\section{MATERIALS AND METHODS}

\section{Xenopus oocytes preparation and maturation in vitro}

Female frogs (Miyamoto et al. 2015) were primed with $50 \mathrm{U}$ of chorionic gonadotropin human (hCG) 5-14 d before the oocyte collection. On the day before oocyte collection, fresh filtered $1 \times$ Modified Barth's Solution ( $1 \times$ MBS) was prepared, and stored at $4^{\circ} \mathrm{C}$. Frogs were anesthetized and euthanized by an overdose of MS222 (Torreilles et al. 2009) followed by decapitation and ovaries were collected and soaked in $1 \times$ MBS. Then ovaries were washed, cut into small pieces $\left(1-2 \mathrm{~cm}^{2}\right)$, and defolliculated with Liberase TL $(0.025 \mathrm{mg} / \mathrm{mL})$ with gently shaking at $23^{\circ} \mathrm{C}$ overnight. The reaction was stopped by adding $1 \times \mathrm{MBS}$, and oocytes were 
rinsed with $1 \times$ MBS for several times and stage VI oocytes were collected using an $800 \mu \mathrm{m}$ filter. The defolliculated oocytes can be used for microinjection assay or maturation. Stage VI oocytes were matured by addition of $3 \mu \mathrm{M}$ progesterone in $1 \times \mathrm{MBS}$ at $21^{\circ} \mathrm{C}$ or $23^{\circ} \mathrm{C}$. Samples were collected at a series of time points (no progesterone, progesterone $90 \mathrm{~min}, \mathrm{GVBD} 0 \mathrm{~min}, \mathrm{GVBD} 30$ min, GVBD 60 min, GVBD 90 min, GVBD 120 min, GVBD 150 min, GVBD $180 \mathrm{~min}$ ) during oogenesis, then flash-frozen in liquid nitrogen and stored at $-80^{\circ} \mathrm{C}$ for: $\mathrm{H} 1$ kinase assay, Tail-seq, analysis of polysomes, and PAT assay. For each time point, five oocytes were used for $\mathrm{H} 1$ kinase assay, Tail-seq and PAT assay, respectively. The oocytes used for TAIL-seq and analysis of polysomes were from the same two frogs, but biological replicates were utilized for PAT assay. For each type of experiment, two biological repeats were performed using two different frogs.

\section{H1 kinase assay}

Five oocytes were homogenized in $100 \mu \mathrm{L}$ of EB (80 mM $\beta$-glycerophosphate, $20 \mathrm{mM}$ EGTA, $15 \mathrm{mM} \mathrm{MgCl}$ ) + leupeptin, pepstatin, cymostatin, and phosphatase inhibitors (type I and type II, Sigma-Aldrich). Lysates were centrifuged at $20,000 \times g$ for $15 \mathrm{~min}$ at $4^{\circ} \mathrm{C}$ and clear extract was collected and stored at $-80^{\circ} \mathrm{C}$. Kinase assays were performed by incubating $5 \mu \mathrm{L}$ of lysate with Histone $\mathrm{H} 1$ and $\gamma-{ }^{32} \mathrm{P}$ ATP in EB for 15 min. Reactions were stopped by the addition of SDS loading buffer and separated using a 15\% SDS-PAGE. Gels were exposed to phosphor-screens for $\sim 3 \mathrm{~h}$ and scanned using a GE Typhoon Imager.

\section{Tail-seq}

Based on $\mathrm{H} 1$ kinase results, oocytes at five time points (no progesterone, progesterone $90 \mathrm{~min}, \mathrm{GVBD} 0 \mathrm{~min}, \mathrm{GVBD} 90 \mathrm{~min}, \mathrm{GVBD}$ $150 \mathrm{~min}$ for repeat 1 or $180 \mathrm{~min}$ for repeat 2) during oogenesis were picked for RNA extraction and RNA-seq library construction. First, oocytes were crushed by pellet pestles in $40 \mu \mathrm{L}$ of XB buffer (100 mM KCl, $0.1 \mathrm{mM} \mathrm{CaCl}_{2}, 1 \mathrm{mM} \mathrm{MgCl}$, $50 \mathrm{mM}$ sucrose, 10 $\mathrm{mM}$ HEPES at $\mathrm{pH}$ 7.7), then $500 \mu \mathrm{L}$ TRIzol was added to extract total RNAs from whole oocytes. Before adding chloroform, yolk and debris were removed by centrifuge $\left(12,000 \mathrm{~g}, 5 \mathrm{~min}\right.$ at $\left.4^{\circ} \mathrm{C}\right)$. The total RNAs in aqueous phase were extracted again using Direct-zol RNA MiniPrep Kit. Tail-seq libraries were constructed based on Harrison et al.'s method (Harrison et al. 2015) with some modifications: Briefly, the $3^{\prime}$ end of RNA was extended by Klenow polymerase exo- at $37^{\circ} \mathrm{C}$ for $1 \mathrm{~h}$, and secondary structures were disrupted by adding $1 \times$ digest buffer and heating at $80^{\circ} \mathrm{C}$ for $10 \mathrm{~min}$. The extended RNAs were fragmented with $0.1 \mathrm{U} / \mu \mathrm{L}$ RNase $\mathrm{T} 1$ at $37^{\circ} \mathrm{C}$ for $15 \mathrm{~min}$, and the reaction was stopped by phenol/chloroform extraction. After binding to streptavidin beads, the cDNAs were 5'-phosphorylated with T4 PNK, ligated with $5^{\prime}$ splinted linker, reverse transcribed using PAT-seq end-extend primer, eluted from streptavidin beads by $30 \mu \mathrm{L}$ of $0.13 \%$ SDS with heating at $100^{\circ} \mathrm{C}$ for $5 \mathrm{~min}$ and then sitting on ice for 2 min. The eluted cDNAs libraries were size selected twice by equal volume of AMPure XP beads (Beckman Coulter, Inc.), and amplified for 14 cycles by NEBNext Ultra II Q5 Master Mix (NEB), NEBNext Universal PCR primer for Illumina (5'-AAT GAT ACG GCG ACC ACC GAG ATC TAC ACT CTT TCC CTA CAC GAC GCT CTT CCG ATC-s-T-3') and NEBNext Index primer for
Illumina (NEB, index\# 9-13 for five libraries in each repeat). Then the amplified CDNAs libraries were purified again by an equal volume of AMPure XP beads (Beckman Coulter, Inc.), and $1 \mu \mathrm{L}$ of eluted libraries were taken for analysis by Bioanalyzer. The size of PAT-seq libraries were 150-1000 bp, with average size from $389 \mathrm{bp}$ to $423 \mathrm{bp}$. For each biological replicate, we mixed the five libraries at a same molar percentage (19.6\%), together with four spikes of $0,8,16$, and 32 As (with index \#8-5) at a same molar percentage (0.1\%) and four spikes of 64,80 , 90, and 118 As (with index \#4-1) at a same molar percentage (0.4\%). The libraries mixtures were run paired-end Illumina HiSeq with two full lanes in rapid mode: 50 cycles reading from the $5^{\prime}$ sq end (P5), and 250 cycles from the 3 'sq end (P7).

\section{Tail-seq sequencing analysis}

Using intensity CIF files and cluster location CLOCS files produced by the Illumina HiSeq instrument in addition to the standard FASTQ files, we extracted flowcell coordinates and intensity of each passing sequencing cluster in the four acquisition channels ( $A, C, G$, and $T$ ). For each sequencing position of read 2 in each library fragment, we calculated the normalized intensities for each of the four channels by normalizing these four values to the total of 1 . We then used these normalized intensities for the positions of the sequencing read to estimate the length of poly(T) tail within each read. Similar to the previous observation (Chang et al. 2014), we observed that in the control samples with the known length of poly $(T)$ tail, the end of the poly $(T)$ tail coincides with a rapid reduction of signal intensity for the $T$ channel and a rapid increase in the signal intensities for non- $T$ channels. To detect these changes along the read length, we developed and optimized a relatively simple and robust computational approach based on the analysis of signal intensities within a sliding window. For each read 2 of the sequencing library, we used a sliding window of $8 \mathrm{bp}$ with the step of $1 \mathrm{bp}$ to calculate the mean normalized intensity for each base type within this window and applied the following rule to determine the end of the poly $(T)$ tail:

$$
T<T_{0} \& \&\left(A>A_{0}\left\|C>C_{0}\right\| G>G_{0}\right),
$$

where $A, T, G, C$ are average signal intensities of each base in the window and $A_{0}, T_{0}, G_{0}, C_{0}$ are the cutoff values of the corresponding intensities. We called the end of the poly(T) tail when this rule was satisfied at least three times among five consecutive positions of the sliding window. The cutoff values $A_{0}$, $T_{0}, G_{0}, C_{0}$ were optimized on the control samples with known lengths of poly $(A)$ tails. We used the following cutoffs: $A_{0}=11$, $T_{0}=45, G_{0}=11, C_{0}=30$.

Read 1 on the opposite end of the library fragment was mapped to the Xenopus laevis transcriptome XL_9.1_v1.8.3.2 using STAR aligner (Dobin et al. 2013). The average poly(A) tail length for each transcript was estimated by calculating the mean length of poly $(T)$ tails among all library fragments with read 1 mapped to a given transcript.

\section{Sucrose density gradient and polysome library preparation}

Sucrose gradients were prepared in $50 \mathrm{mM}$ Tris pH 7.5, $250 \mathrm{mM}$ $\mathrm{KCl}, 25 \mathrm{mM} \mathrm{MgCl}_{2}$. Gradients were poured in steps and frozen in 
liquid nitrogen between steps. Gradients were stored at $-80^{\circ} \mathrm{C}$ and thawed overnight at $4^{\circ} \mathrm{C}$ before use. Oocytes $(\sim 100)$ from each stage were incubated in polysome buffer $(20 \mathrm{mM}$ Tris $\mathrm{pH}$ $7.5,0.5 \% \mathrm{NP}-40,300 \mathrm{mM} \mathrm{KCl}, 2 \mathrm{mM} \mathrm{MgCl} 2$ with $0.1 \mathrm{mg} / \mathrm{mL}$ cycloheximide) for $5 \mathrm{~min}$ at room temperature. Oocytes were then allowed to settle and buffer was removed. Oocytes were crushed with a pestle and centrifuged at $17,000 \times \mathrm{g}$ for $15 \mathrm{~min}$ at $4^{\circ} \mathrm{C}$. The cytoplasmic layer was removed for use in sucrose gradients. Twenty-five microliters of the cytoplasmic extract was diluted with $250 \mu \mathrm{L}$ of PB containing cycloheximide and loaded onto a thawed sucrose gradient. Gradients were centrifuged at 39,000 rpm using a SW41 rotor for $2 \mathrm{~h}$ at $4^{\circ} \mathrm{C}$. Tubes were pierced using a Brandel tube piercer and fractionated by upward displacement using a GE Akta FPLC. Five hundred microliter fractions spanning the entire gradient were collected and stored at $-80^{\circ} \mathrm{C}$.

Total RNA was purified from each fraction using TRIzol. RNA samples from mRNP, monosome, and polyribosomes were pooled and digested with RQ1 DNase. DNase-treated RNA was cleaned up using the Zymo Clean \& Concentrate Kit. One hundred nanograms of total RNA from human RPE-1 cells was added to each sample and poly(A) RNA was purified using Exiqon LNA dT Kit. Poly(A) RNA was then used as input for the NEBNext Ultra Directional Library Prep Kit for Illumina according to the manufacturer's instructions. Libraries were PAGE purified (we selected 250-600 bp range) and sequenced on an Illumina HiSeq.

\section{Polysome sequence alignment and analysis}

Reads were collapsed into unique sequences using a custom Perl script (Schwarz and Blower 2014). Unique reads were aligned to Xenbase mRNA sequences using Bowtie2 or to the Xenopus laevis 9.2 genome release using TopHat2 (Trapnell et al. 2012). In addition, unique reads were aligned to human RefSeq RNAs using Bowtie2. Reads were counted against mRNAs using a custom Perl script (Schwarz and Blower 2014) or the cuffdiff function of Cufflinks. FPKM values for all human genes detected at a FPKM of $>10$ were used to normalize FPKM values between libraries. For each sucrose gradient we used normalized FPKM values to calculate the percentage of mRNA present in the polysome fraction of the gradient. Polysome percentages were used to analyze changes in mRNA translation during oocyte maturation. We analyzed mRNAs that could be detected at a FPKM $>1$ in all samples from both biological replicates. All sequences from polysome gradients and PAS-seq were deposited in GEO with accession number GSE134537.

\section{RNA degradation analysis}

To measure transcript stability during oocyte maturation we used reads generated from our sucrose gradient experiments. We merged fastq files from each gradient fraction for each time point to create a "total RNA" file for each time point. We then used TopHat2 and Cufflinks to align and count these reads against the $X$. laevis genome. We searched for reproducibly up- and down-regulated RNAs using STEM software.

\section{PAT assay and TVN PCR}

Based on $\mathrm{H} 1$ kinase results, oocytes from five time points (no progesterone, progesterone $90 \mathrm{~min}$, GVBD $0 \mathrm{~min}$, GVBD $120 \mathrm{~min}$ for repeat1 or 150 min for repeat 2, GVBD $180 \mathrm{~min}$ ) during oogenesis were selected and total RNA was purified using TRlzol. Using USB Poly(A) Tail-Length Assay Kit (Affymetrix, USB), $0.5 \mu \mathrm{g}$ total RNAs were used for each $10 \mu \mathrm{L}$ of $\mathrm{G} / \mathrm{I}$ tailing reaction, then the reaction was stopped and $7.5 \mu \mathrm{L}$ were reverse transcribed, and $2.5 \mu \mathrm{L}$ were used as a negative control with no reverse transcription. NEBNext Ultra II Q5 Master Mix (NEB) was used to amplify diluted poly $(G / l)$ tailed cDNAs with PAT Universal Reverse Primer (5'-GGTAATACGACTCACTATAGCGAGACCCCCCCCCCTT-3') and forward gene-specific primer. TVN PCR was used as a control of PAT assay: $2.5 \mu \mathrm{g}$ total RNAs per $20 \mu \mathrm{L}$ reaction were reversed transcribed at $50^{\circ} \mathrm{C}$ for $1 \mathrm{~h}$, using TVN PCR reverse primer (5'-CAAGCAGAAGACGGCATACGATTTTTTTTTTTTTTTTTVN-3') and SuperScript III Reverse Transcriptase (Invitrogen), and then the reaction was stopped by heating at $70^{\circ} \mathrm{C}$ for $15 \mathrm{~min}$. To reduce non-specific amplification, touch-down PCR programs $\left(98^{\circ} \mathrm{C}\right.$, $30 \mathrm{sec} ;\left[98^{\circ} \mathrm{C}, 10 \mathrm{sec}, 72^{\circ} \mathrm{C}, 75 \mathrm{sec}\right]$ seven cycles, $-1^{\circ} \mathrm{C}$ each cycle; $\left[98^{\circ} \mathrm{C}, 10 \mathrm{sec} ; 65^{\circ} \mathrm{C}, 75 \mathrm{sec}\right] 23$ cycles, $65^{\circ} \mathrm{C}, 5 \mathrm{~min} ; 12^{\circ} \mathrm{C}$, end) were used to amplify cDNAs from PAT assay and TVN PCR. Finally, PCR products from PAT assay and TVN PCR were run on the same native TBE gels and stained with SYBR Gold Nucleic Acid Gel Stain (Invitrogen) for analysis.

\section{PAS-seq library preparation}

Total RNAs extracted from stage VI oocytes (no PG) were used for preparation of PAS-seq libraries from three biological replicates. However, during library sequencing barcode reading failed and we could not separate the three libraries, therefore they were treated as a single replicate. PAS-seq primers are listed below:
HITS-3 (5'-ACA CTC TTT CCC TAC ACG ACG CTC TTC CGA TCT TTT TTT TTT TTT TTT TTT TVN NN-3'),
HITS-5 (5'-CGG TCT CGG CAT TCC TGC TGA ACC GCT CTT CCG ATC TrGrG rG-3'),
PA1.0 (5'-AAT GAT ACG GCG ACC ACC GAG ATC TAC ACT CTT TCC CTA CAC GAC GCT CTT CCG ATC TTT TTT CTT TTT TCT TTT TT-3'),
PAlndex1 (5'-CAA GCA GAA GAC GGC ATA CGA GAT ATC ACG CGG TCT CGG CAT TCC TGC TGA ACC GCT CTT CCG ATC T-3'),
PAlndex2 (5'-CAA GCA GAA GAC GGC ATA CGA GAT CGA TGT CGG TCT CGG CAT TCC TGC TGA ACC GCT CTT CCG ATC T-3'),
PAlndex3 (5'-CAA GCA GAA GAC GGC ATA CGA GAT TTA GGC CGG TCT CGG CAT TCC TGC TGA ACC GCT CTT CCG ATC T-3'),
Sequencing primer (5'-ACA CTC TTT CCC TAC ACG ACG CTC TTC CGA TCT TTT TTC TTT TTT CTT TTT T-3').

\section{PAS-seq sequence alignment and data analysis}

Reads from two HiSeq lanes were combined into a single fastq file and collapsed into unique reads. Linker sequences were removed using the FASTX-Toolkit keeping all trimmed reads $30 \mathrm{nt}$ or longer. Reads were aligned to $X$. laevis 9.2 genome assembly using Bowtie2. Aligned reads were filtered to retail alignments that contained two or more nontemplated As, originating from the 
poly(A) tail. We then used Yodel peak calling software (https ://github.com/LancePalmerStJude/YODEL) to convert aligned PAS-seq reads into peaks. Yodel was used with default settings and required a minimum peak height of 4 . All PAS-seq reads and poly(A) site calls are deposited in GEO under accession GSE134537.

\section{Prediction of $3^{\prime}$ UTRs based on PAS-seq}

We first predicted 42,859 poly(A) sites located at genome, based on PAS-seq read peaks called using Yodel software (reads $\geq 4$ ). We used the following criteria: If reads are aligned to the + strand of genome, poly(A) site should be at the $5^{\prime}$-most of peak aligned region; if reads are aligned to the - strand of genome, poly $(A)$ site should be at the $3^{\prime}$-most of peak aligned region. Next, we determined that 41,982 poly(A) sites intersected with gene models "XENLA_9.2_Xenbase.gff3," using the following criteria: (1) Poly(A) site should be located at the same chromosome or scaffold as the gene. (2) The alignment of poly(A) site to the genome should be located at the opposite strand of the gene. (3) The poly (A) site should be located in the range from the start of the gene to $5 \mathrm{~kb}$ downstream from the gene. Then, 5359 poly(A) sites that have six or more consecutive A's or seven or more A's in the $10 \mathrm{nt}$ immediately downstream from the poly(A) junction were removed as they are likely due to internal priming (Shepard et al. 2011). For each poly(A) site in each gene, we calculated the ratio using the formula that most read number is divided by other read number, respectively. Then we only kept 25,735 poly(A) sites with the ratio $\leqq 4$ for the following analysis. Using gene model "XENLA_9.2_Xenbase.gff3," we found 46,582 X. laevis genes with stop codons, and picked 43,793 genes with unique stop codons to locate $\operatorname{poly}(A)$ sites and predict their corresponding 3'UTRs.

For the $3^{\prime} U T R$ prediction, we chose 15,805 poly(A) sites after the unique stop codon, and only one mRNA with the most annotated exons after the stop codon for each gene.

$3^{\prime}$ UTRs are from the first nucleotide after the stop codon to the poly(A) site on exons. 15,805 of 3'UTRs are predicted by the following criteria: (1) For genes on + strand, poly(A) site is after stop codon with two conditions: (1.1) No exons are between poly(A) site and stop codon. 3'UTR sequence is all nucleotides between (stop codon, poly(A) site]. (1.2) Exons are between poly(A) site and stop codon, also with two conditions: (1.2.1) poly(A) site is between the two ends of an exon. $3^{\prime}$ UTR sequence is all exon nucleotides between (stop codon, poly(A) site]. (1.2.2) poly(A) site is between two exons. $3^{\prime} U T R$ sequence is all exon nucleotides between (stop codon, $\operatorname{poly}(A)$ site], together with the intron nucleotides between [last valid exon above, poly(A) site]. (2) For genes on - strand, poly $(A)$ site is before stop codon with two conditions: (2.1) No exons are between poly(A) site and stop codon. $3^{\prime} U T R$ is reverse complement of all nucleotides between [poly(A) site, stop codon). (2.2) Exons are between poly(A) site and stop codon with two conditions. (2.2.1) poly(A) site is between two ends of an exon. $3^{\prime}$ UTR is reverse complement of all exon nucleotides between [poly(A) site, stop codon). (2.2.2) poly(A) site is between two exons. $3^{\prime}$ UTR is reverse complement of the intron nucleotide between [poly(A) site, first valid exon above], together with all exon nucleotides between [poly(A) site, stop codon). "[" and "]" means being included, "(" and ")" means not being in- cluded. Finally, we picked 10,600 of 3'UTRs with the most read numbers for each gene for future analysis.

\section{Distributions of poly(A) sites on genes based on PAS-seq}

For 25,735 poly(A) sites located on the gene model, we obtained 15,805 poly $(A)$ sites after and 6730 poly $(A)$ sites before the stop codon of mRNAs used for 3'UTRs prediction. We then picked 2789 genes with multiple stop codons, and located 3001 poly (A) sites on these genes. The remaining 199 poly(A) sites are found on genes with no stop codon (MT genes, IncRNA genes etc.). For the 6730 poly(A) sites before the stop codon, we located them on 5' UTRs, CDSs, and introns. Briefly, for each poly(A) site, we first got the exon and CDS information of their corresponding mRNAs in "XENLA_9.2_Xenbase.gff3." Then we arranged exons and CDSs in order from small position to big, based on their left and right end value. Next, we defined introns and 5'UTRs using the following criteria: Introns of all nucleotides between (right end of exon, left end of next exon). For mRNAs on + strand, $5^{\prime}$ UTRs are all nucleotides between [left end of the first exon, the left end of the first CDS); for mRNAs on - strand, 5'UTRs are all nucleotides between (right end of last cds, right end of last exon]. "[" and "]" means being included, "(" and ")" means not being included. Finally, we located 402 poly(A) sites on 5'UTRs, 4426 on CDSs and 1844 on introns. There are 58 remaining poly(A) sites with 57 on mRNAs, which are not the ones used for $3^{\prime}$ UTRs prediction, and one on mRNAs with no stop codon.

\section{Sequence motif analysis}

To analyze enriched motifs, we used 3'UTR sequences predicted from our PAS-seq data. For mRNAs with multiple PAS sites, we analyzed the sequence supported by the largest number of PAS reads (11,055 sequences). To analyze hexamer composition, we calculated that density (\# of motif occurrences per kb) of all hexamers in various clusters of UTRs (from Figs. 1, 2, and 3) and all transcripts. We then calculated hexamer enrichment by subtracting the mean hexamer density in all transcripts from the mean density in a cluster of transcripts. For each hexamer, we also calculated a Z-score for motif density and calculated the percentage of $3^{\prime}$ UTRs that contained the motif. We repeated this analysis with motif lengths from 4 nt to $8 \mathrm{nt}$ and observed similar results.

To analyze the relative position of U-rich motifs in $3^{\prime} U T R s$, we identified all sequences that contained a match to a canonical polyadenylation sequence (AAUAAA or AUUAAA) and retained the most $3^{\prime}$ polyadenylation location. We then calculated the distance of all matches to the top 13 U-rich motifs to the most $3^{\prime}$ polyadenylation signal for various clusters of RNAs and for all transcripts.

\section{Prepare mRNAs for microinjection}

Firefly luciferase CDS was cloned into PCR2.1-TOPO vector, using EcoRV (5'EcoRV-FF Luciferase: TCAGGGATATCgccgcC CCCATGGAAGACGCCAAAAACAT) and BamHI (3'BamHI-FF Luciferase: CGCGGATCCTTACACGGCGAT CTTTCCGCCCTT) restriction sites. Then spc25.L 3'UTR (gBlocks Gene Fragment 
from IDT) was cloned after the firefly luciferase CDS, using BamHI and HindllI restriction sites, and $5 \times$ In-Fusion HD Enzyme Premix (TaKaRa). Based on Firefly luciferase- spc25.L 3'UTR - pCR2.1TOPO plasmid, four spc25.L 3'UTR mutations were made by Q5 Site-Directed Mutagenesis Kit (NEB, E0554).

Our former renilla luciferase on PCR2.1-TOPO plasmid (pMB342) does not have a poly(A) tail. To increase the stability of renilla luciferase mRNA, we lengthened its $3^{\prime}$ UTRs and also added an 80 As poly(A) tail on its plasmid (Gebauer et al. 1994; Piqué et al. 2006), using the following method: First, we prepared a dsDNA fragment (including $80 \mathrm{As}$ ) by annealing its sense and antisense oligoes ( $20 \mu \mathrm{M}$ for each) in nuclease-free water $\left(95^{\circ} \mathrm{C}\right.$, $5 \mathrm{~min}$; then decreased the temperature by $0.1^{\circ} \mathrm{C} / \mathrm{sec}$ until $12^{\circ} \mathrm{C}$ ). Then we cloned the dsDNA fragment into our pMB342 plasmid, using Bam $\mathrm{HI}$ and Sacl restriction sites, and $5 \times \mathrm{In}$ Fusion HD Enzyme Premix (TaKaRa).

Next, we prepared DNA templates for in vitro transcription. For firefly luciferase-spc25.L 3'UTRs, we added 12 As poly(A) tails to their DNA templates, by PCR amplification (NEBNext Ultra II Q5 Master Mix, NEB) and then Dpnl digestion to remove the plasmids. For renilla luciferase-3'UTR, we made its template by HindIII digestion after its 80 As poly(A) tail. The corresponding mRNAs were prepared by HiScribe T7 Quick High Yield RNA Synthesis Kit (NEB, E2050S), and capped by Vaccinia Capping System (NEB, M2080S). The capped mRNAs were then purified by Direct-zol RNA MiniPrep Kits (Zymo Research). Finally, we made the hybrid reporter mRNA stocks $(0.05 \mathrm{pmol} / \mu \mathrm{L}$ of capped firefly luciferase-spc25.L 3'UTRs-A12 or renilla luciferase-3'UTRA80), and stored them at $-80^{\circ}$.

\section{Microinjection and luciferase reporter assay}

The microinjection and luciferase reporter assay generally followed the method from Piqué et al with some modifications (Piqué et al. 2006, 2008). After using an $800 \mu \mathrm{m}$ filter to collect the stage $\mathrm{VI}$ oocytes, we picked the defolliculated oocytes into a new plate containing $1 \times \mathrm{MBS}$ for microinjection. The hybrid reporter mRNAs stocks were heated at $70^{\circ} \mathrm{C}$ for $7 \mathrm{~min}$ and then put on ice to eliminate the secondary structures. Before microinjection, we diluted the hybrid reporter mRNA stocks to 0.0002 $\mathrm{pmol} / \mu \mathrm{L}$ for each mRNA. Twenty-five nanoliters of diluted hybrid reporter mRNAs (0.005 fmol of each mRNA) were injected into each oocyte. After microinjection of each sample, oocytes were set at $21^{\circ} \mathrm{C}$ for $5 \mathrm{~min}$, and then split into two plates: One is for no progesterone treatment ( 1 ×MBS only) ("NoPG", 40 oocytes), the other is for maturation ( $3 \mu \mathrm{M}$ progesterone in $1 \times$ MBS) ( 60 oocytes). The two plates were placed at $23^{\circ} \mathrm{C}$ for maturation. For each sample, GVBD and "NoPG" oocytes were collected at the same time (five oocytes/repeat, three repeats) (de Moor and Richter 1997), when GVBD happened in more than $50 \%$ oocytes. Then $1 \times$ MBS buffer in each tube was removed as much as possible. Tubes were frozen in liquid nitrogen and stored at $-80^{\circ} \mathrm{C}$. Two biological repeats were performed using two different frogs. For luciferase reporter assay, oocytes were lysated in $50 \mu \mathrm{L} 1 \times \mathrm{MBS}(10 \mu \mathrm{L} 1 \times \mathrm{MBS} /$ each oocyte) by pipetting up and down through a $200-\mu \mathrm{L}$ tip. Then the samples were cleared by centrifugation $\left(12,000 \mathrm{~g}, 5 \mathrm{~min}\right.$, at $\left.4^{\circ} \mathrm{C}\right), 40 \mu \mathrm{L}$ clear supernatant was transferred into a new tube, and $10 \mu \mathrm{L}$ supernatant was used for the following assay. Fifty microliters of LAR II and $50 \mu \mathrm{L}$ Stop \&
Glo buffer (Promega, E1910) were used to measure firefly and renilla luciferase activity.

\section{DATA DEPOSITION}

All raw and processed sequencing data generated in this study have been submitted to the NCBI Gene Expression Omnibus (GEO; http://www.ncbi.nlm.nih.gov/geo/) under accession number GSE134537.

\section{SUPPLEMENTAL MATERIAL}

Supplemental material is available for this article.

\section{ACKNOWLEDGMENTS}

We thank Ulandt Kim and the Molecular Biology sequencing core for help with optimization of the Tail-seq procedure, Adrian Salic for the gift of Sgo1 antibodies, and Judith Sharp and Carlos Perea-Resa for comments on the manuscript. This work was funded by a grant to M.D.B. from the National Institutes of Health/ National Institute of General Medical Sciences (GM108548) and by NIH/National Institute of Diabetes and Digestive and Kidney Diseases P30 DK040561 to R.I.S.

Received September 3, 2019; accepted December 30, 2019.

\section{REFERENCES}

Afroz T, Skrisovska L, Belloc E, Guillen-Boixet J, Mendez R, Allain FH. 2014. A fly trap mechanism provides sequence-specific RNA recognition by CPEB proteins. Genes Dev 28: 1498-1514. doi:10 $.1101 / \mathrm{gad} .241133 .114$

Bajak EZ, Hagedorn CH. 2008. Efficient 5' cap-dependent RNA purification: use in identifying and studying subsets of RNA. Methods Mol Biol 419: 147-160. doi:10.1007/978-1-59745-033-1_10

Beilharz TH, Preiss T. 2007. Widespread use of poly(A) tail length control to accentuate expression of the yeast transcriptome. RNA 13: 982-997. doi:10.1261/rna.569407

Blower MD, Jambhekar A, Schwarz DS, Toombs JA. 2013. Combining different mRNA capture methods to analyze the transcriptome: analysis of the Xenopus laevis transcriptome. PLoS One 8: e77700. doi:10.1371/journal.pone.0077700

Chang H, Lim J, Ha M, Kim VN. 2014. TAlL-seq: genome-wide determination of poly(A) tail length and $3^{\prime}$ end modifications. Mol Cell 53: 1044-1052. doi:10.1016/j.molcel.2014.02.007

Chen J, Melton C, Suh N, Oh JS, Horner K, Xie F, Sette C, Blelloch R, Conti M. 2011. Genome-wide analysis of translation reveals a critical role for deleted in azoospermia-like (Dazl) at the oocyte-to-zygote transition. Genes Dev 25: 755-766. doi:10.1101/gad .2028911

Collier B, Gorgoni B, Loveridge C, Cooke HJ, Gray NK. 2005. The DAZL family proteins are PABP-binding proteins that regulate translation in germ cells. EMBO J 24: 2656-2666. doi:10.1038/sj .emboj.7600738

Curanovic D, Cohen M, Singh I, Slagle CE, Leslie CS, Jaffrey SR. 2013. Global profiling of stimulus-induced polyadenylation in cells using a poly(A) trap. Nat Chem Biol 9: 671-673. doi:10.1038/nchembio .1334 
de Moor CH, Richter JD. 1997. The Mos pathway regulates cytoplasmic polyadenylation in Xenopus oocytes. Mol Cell Biol 17: 64196426. doi:10.1128/MCB.17.11.6419

Diaz-Munoz MD, Kiselev VY, Le Novere N, Curk T, Ule J, Turner M. 2017. Tia1 dependent regulation of mRNA subcellular location and translation controls p53 expression in B cells. Nat Commun 8: 530. doi:10.1038/s41467-017-00454-2

Dobin A, Davis CA, Schlesinger F, Drenkow J, Zaleski C, Jha S, Batut P, Chaisson M, Gingeras TR. 2013. STAR: ultrafast universal RNA-seq aligner. Bioinformatics 29: 15-21. doi:10.1093/bioinformatics/ bts635

Dominguez D, Freese $P$, Alexis MS, Su A, Hochman M, Palden $T$, Bazile C, Lambert NJ, Van Nostrand EL, Pratt GA, et al. 2018. Sequence, structure, and context preferences of human RNA binding proteins. Mol Cell 70: 854-867.e859. doi:10.1016/j.molcel .2018.05.001

Eichhorn SW, Subtelny AO, Kronja I, Kwasnieski JC, Orr-Weaver TL, Bartel DP. 2016. mRNA poly(A)-tail changes specified by deadenylation broadly reshape translation in Drosophila oocytes and early embryos. Elife 5: e16955. doi:10.7554/eLife.16955

Eliscovich C, Peset I, Vernos I, Méndez R. 2008. Spindle-localized CPE-mediated translation controls meiotic chromosome segregation. Nat Cell Biol 10: 858-865. doi:10.1038/ncb1746

Eliseeva IA, Lyabin DN, Ovchinnikov LP. 2013. Poly(A)-binding proteins: structure, domain organization, and activity regulation. Biochemistry (Mosc) 78: 1377-1391. doi:10.1134/S0006297913 130014

Ernst J, Bar-Joseph Z. 2006. STEM: a tool for the analysis of short time series gene expression data. BMC Bioinformatics 7: 191. doi:10 .1186/1471-2105-7-191

Gebauer F, Xu W, Cooper GM, Richter JD. 1994. Translational control by cytoplasmic polyadenylation of c-mos mRNA is necessary for oocyte maturation in the mouse. EMBO J 13: 5712-5720. doi:10 .1002/j.1460-2075.1994.tb06909.x

Gruber AJ, Zavolan M. 2019. Alternative cleavage and polyadenylation in health and disease. Nat Rev Genet 20: 599-614. doi:10 .1038/s41576-019-0145-z

Hannak E, Heald R. 2006. Investigating mitotic spindle assembly and function in vitro using Xenopus laevis egg extracts. Nat Protoc 1: 2305-2314. doi:10.1038/nprot.2006.396

Harland RM, Grainger RM. 2011. Xenopus research: metamorphosed by genetics and genomics. Trends Genet 27: 507-515. doi:10 .1016/j.tig.2011.08.003

Harrison PF, Powell DR, Clancy JL, Preiss T, Boag PR, Traven A, Seemann T, Beilharz TH. 2015. PAT-seq: a method to study the integration of 3'-UTR dynamics with gene expression in the eukaryotic transcriptome. RNA 21: 1502-1510. doi:10.1261/rna.048355 .114

Hassold T, Hunt P. 2001. To err (meiotically) is human: the genesis of human aneuploidy. Nat Rev Genet 2: 280-291. doi:10.1038/ 35066065

Hu W, Li S, Park JY, Boppana S, Ni T, Li M, Zhu J, Tian B, Xie Z, Xiang M. 2017. Dynamic landscape of alternative polyadenylation during retinal development. Cell Mol Life Sci 74: 1721-1739. doi:10.1007/s00018-016-2429-1

Huang da W, Sherman BT, Lempicki RA. 2009. Systematic and integrative analysis of large gene lists using DAVID bioinformatics resources. Nat Protoc 4: 44-57. doi:10.1038/nprot.2008.211

Kronja I, Yuan B, Eichhorn SW, Dzeyk K, Krijgsveld J, Bartel DP, OrrWeaver TL. 2014. Widespread changes in the posttranscriptional landscape at the Drosophila oocyte-to-embryo transition. Cell Rep 7: 1495-1508. doi:10.1016/j.celrep.2014.05.002

Kuge $H$, Inoue A. 1992. Maturation of Xenopus laevis oocyte by progesterone requires poly(A) tail elongation of mRNA. Exp Cell Res 202: 52-58. doi:10.1016/0014-4827(92)90403-U
Lambert NJ, Robertson AD, Burge CB. 2015. RNA bind-n-seq: measuring the binding affinity landscape of RNA-binding proteins. Methods Enzymol 558: 465-493. doi:10.1016/bs.mie.2015.02 .007

Lim J, Lee M, Son A, Chang H, Kim VN. 2016. mTAIL-seq reveals dynamic poly $(A)$ tail regulation in oocyte-to-embryo development. Genes Dev 30: 1671-1682. doi:10.1101/gad.284802.116

Mendez R, Richter JD. 2001. Translational control by CPEB: a means to the end. Nat Rev Mol Cell Biol 2: 521-529. doi:10.1038/ 35080081

Miyamoto K, Simpson D, Gurdon JB. 2015. Manipulation and in vitro maturation of Xenopus laevis oocytes, followed by intracytoplasmic sperm injection, to study embryonic development. J Vis Exp: e52496. doi:10.3791/52496

Morgan M, Much C, DiGiacomo M, Azzi C, Ivanova I, Vitsios DM, Pistolic J, Collier P, Moreira PN, Benes V, et al. 2017. mRNA 3' uridylation and poly $(A)$ tail length sculpt the mammalian maternal transcriptome. Nature 548: 347-351. doi:10.1038/nature23318

Nagaoka SI, Hassold TJ, Hunt PA. 2012. Human aneuploidy: mechanisms and new insights into an age-old problem. Nat Rev Genet 13: 493-504. doi:10.1038/nrg3245

Park JE, Yi H, Kim Y, Chang H, Kim VN. 2016. Regulation of poly(A) tail and translation during the somatic cell cycle. Mol Cell 62: 462471. doi:10.1016/j.molcel.2016.04.007

Piqué M, López JM, Méndez R. 2006. Cytoplasmic mRNA polyadenylation and translation assays. Methods Mol Biol 322: 183-198. doi:10.1007/978-1-59745-000-3_13

Piqué M, López JM, Foissac S, Guigó R, Méndez R. 2008. A combinatorial code for CPE-mediated translational control. Cell 132: 434448. doi:10.1016/j.cell.2007.12.038

Prescott DM, Bender MA. 1962. Synthesis of RNA and protein during mitosis in mammalian tissue culture cells. Exp Cell Res 26: 260268. doi:10.1016/0014-4827(62)90176-3

Pyronnet S, Dostie J, Sonenberg N. 2001. Suppression of cap-dependent translation in mitosis. Genes Dev 15: 2083-2093. doi:10 $.1101 / \mathrm{gad} .889201$

Ray D, Kazan H, Cook KB, Weirauch MT, Najafabadi HS, Li X, Gueroussov S, Albu M, Zheng H, Yang A, et al. 2013. A compendium of RNA-binding motifs for decoding gene regulation. Nature 499: 172-177. doi:10.1038/nature12311

Revenkova E, Herrmann K, Adelfalk C, Jessberger R. 2010. Oocyte cohesin expression restricted to predictyate stages provides full fertility and prevents aneuploidy. Curr Biol 20: 1529-1533. doi:10.1016/j.cub.2010.08.024

Richter JD. 2007. CPEB: a life in translation. Trends Biochem Sci 32: 279-285. doi:10.1016/j.tibs.2007.04.004

Richter JD, Lasko P. 2011. Translational control in oocyte development. Cold Spring Harb Perspect Biol 3: a002758. doi:10.1101/ cshperspect.a002758

Schoenberg DR, Maquat LE. 2012. Regulation of cytoplasmic mRNA decay. Nat Rev Genet 13: 246-259. doi:10.1038/nrg3160

Schwarz DS, Blower MD. 2014. The calcium-dependent ribonuclease XendoU promotes ER network formation through local RNA degradation. J Cell Biol 207: 41-57. doi:10.1083/jcb.201406037

Sheets MD, Fox CA, Hunt T, Vande Woude G, Wickens M. 1994. The $3^{\prime}$-untranslated regions of c-mos and cyclin mRNAs stimulate translation by regulating cytoplasmic polyadenylation. Genes Dev 8: 926-938. doi:10.1101/gad.8.8.926

Shepard PJ, Choi EA, Lu J, Flanagan LA, Hertel KJ, Shi Y. 2011. Complex and dynamic landscape of RNA polyadenylation revealed by PAS-Seq. RNA 17: 761-772. doi:10.1261/rna.2581711

Stebbins-Boaz B, Hake LE, Richter JD. 1996. CPEB controls the cytoplasmic polyadenylation of cyclin, Cdk2 and c-mos mRNAs and is necessary for oocyte maturation in Xenopus. EMBO J 15: 2582 2592. doi:10.1002/j.1460-2075.1996.tb00616.x 
Subtelny AO, Eichhorn SW, Chen GR, Sive H, Bartel DP. 2014. Poly(A)tail profiling reveals an embryonic switch in translational control. Nature 508: 66-71. doi:10.1038/nature13007

Tachibana-Konwalski K, Godwin J, van der Weyden L, Champion L, Kudo NR, Adams DJ, Nasmyth K. 2010. Rec8-containing cohesin maintains bivalents without turnover during the growing phase of mouse oocytes. Genes Dev 24: 2505-2516. doi:10.1101/gad .605910

Tanenbaum ME, Stern-Ginossar N, Weissman JS, Vale RD. 2015. Regulation of mRNA translation during mitosis. Elife 4: e07957. doi:10.7554/eLife.07957

Tian B, Manley JL. 2017. Alternative polyadenylation of mRNA precursors. Nat Rev Mol Cell Biol 18: 18-30. doi:10.1038/nrm.2016.116

Torreilles SL, McClure DE, Green SL. 2009. Evaluation and refinement of euthanasia methods for Xenopus laevis. J Am Assoc Lab Anim Sci 48: 512-516.

Trapnell C, Roberts A, Goff L, Pertea G, Kim D, Kelley DR, Pimentel H, Salzberg SL, Rinn JL, Pachter L. 2012. Differential gene and transcript expression analysis of RNA-seq experiments with TopHat and Cufflinks. Nat Protoc 7: 562-578. doi:10.1038/nprot.2012 .016

Ulitsky I, Shkumatava A, Jan CH, Subtelny AO, Koppstein D, Bell GW, Sive H, Bartel DP. 2012. Extensive alternative polyadenylation during zebrafish development. Genome Res 22: 2054-2066. doi:10 $.1101 /$ gr.139733.112

Varnum SM, Wormington WM. 1990. Deadenylation of maternal mRNAs during Xenopus oocyte maturation does not require specific cis-sequences: a default mechanism for translational control. Genes Dev 4: 2278-2286. doi:10.1101/gad.4.12b.2278

Vastenhouw NL, Cao WX, Lipshitz HD. 2019. The maternal-to-zygotic transition revisited. Development 146: dev161471. doi:10.1242/ dev. 161471

Vejnar CE, Abdel Messih M, Takacs CM, Yartseva V, Oikonomou P, Christiano R, Stoeckius M, Lau S, Lee MT, Beaudoin JD, et al. 2019. Genome wide analysis of 3' UTR sequence elements and proteins regulating mRNA stability during maternal-to-zygotic transition in zebrafish. Genome Res 29: 1100-1114. doi:10 $.1101 /$ gr.245159.118

Voeltz GK, Steitz JA. 1998. AUUUA sequences direct mRNA deadenylation uncoupled from decay during Xenopus early development. Mol Cell Biol 18: 7537-7545. doi:10.1128/MCB.18.12 .7537

Wang T, Wang H, Cai D, Gao Y, Zhang H, Wang Y, Lin C, Ma L, Gu LL. 2017. Comprehensive profiling of rhizome-associated alternative splicing and alternative polyadenylation in moso bamboo (Phyllostachys edulis). Plant J 91: 684-699. doi:10.1111/tpj.13597

Weill L, Belloc E, Bava FA, Méndez R. 2012. Translational control by changes in poly $(A)$ tail length: recycling mRNAs. Nat Struct Mol Biol 19: 577-585. doi:10.1038/nsmb.2311

Weill L, Belloc E, Castellazzi CL, Méndez R. 2017. Musashi 1 regulates the timing and extent of meiotic mRNA translational activation by promoting the use of specific CPEs. Nat Struct Mol Biol 24: 672681. doi:10.1038/nsmb.3434

Wu X, Bartel DP. 2017. Widespread influence of 3 '-end structures on mammalian mRNA processing and stability. Cell 169: 905-917. e911. doi:10.1016/j.cell.2017.04.036

Zhang J, Kong L, Guo S, Bu M, Guo Q, Xiong Y, Zhu N, Qiu C, Yan X, Chen $Q$, et al. 2016. hnRNPs and ELAVL1 cooperate with uORFs to inhibit protein translation. Nucleic Acids Res 45: 2849-2864. doi:10.1093/nar/gkw991

Zhou Q, Fu H, Yang D, Ye C, Zhu S, Lin J, Ye W, Ji G, Ye X, Wu X, et al. 2019. Differential alternative polyadenylation contributes to the developmental divergence between two rice subspecies, japonica and indica. Plant J 98: 260-276. doi:10.1111/tpj.14209

Ziegler D, Masui Y. 1976. Control of chromosome behavior in amphibian oocytes. II. The effect of inhibitors of RNA and protein synthesis on the induction of chromosome condensation in transplanted brain nuclei by oocyte cytoplasm. J Cell Biol 68: 620-628. doi:10.1083/jcb.68.3.620 

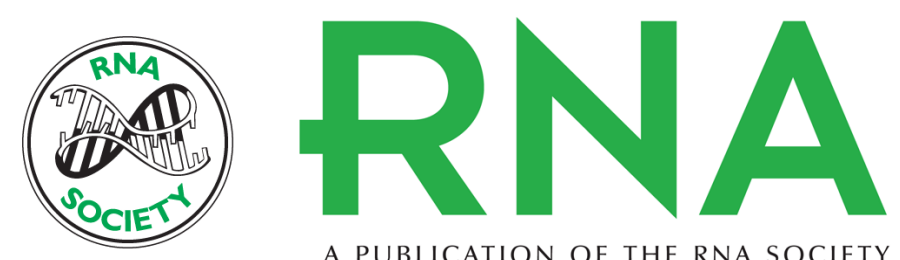

A PUBLICATION OF THE RNA SOCIETY

\section{Genome-wide analysis identifies cis-acting elements regulating mRNA polyadenylation and translation during vertebrate oocyte maturation}

Fei Yang, Wei Wang, Murat Cetinbas, et al.

RNA 2020 26: 324-344 originally published online January 2, 2020

Access the most recent version at doi:10.1261/rna.073247.119

\section{Supplemental http://rnajournal.cshlp.org/content/suppl/2020/01/02/rna.073247.119.DC1 \\ Material}

References This article cites 64 articles, 19 of which can be accessed free at: http://rnajournal.cshlp.org/content/26/3/324.full.html\#ref-list-1

Creative This article is distributed exclusively by the RNA Society for the first 12 months after the Commons

License full-issue publication date (see http://rnajournal.cshlp.org/site/misc/terms.xhtml). After 12 months, it is available under a Creative Commons License (Attribution-NonCommercial 4.0 International), as described at http://creativecommons.org/licenses/by-nc/4.0/.

Email Alerting Receive free email alerts when new articles cite this article - sign up in the box at the Service top right corner of the article or click here.

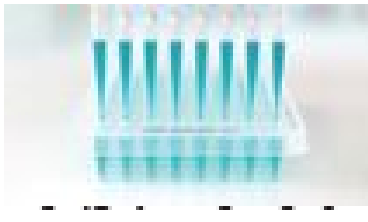

Providing Precise Solutions for your research.

To subscribe to $R N A$ go to:

http://rnajournal.cshlp.org/subscriptions 